\title{
Luminous supersoft X-ray sources
}

\author{
O. M. Bitzaraki ${ }^{1}$, H. Rovithis-Livaniou ${ }^{1}$, C. A. Tout ${ }^{2}$, and E. P. J. van den Heuvel ${ }^{3}$ \\ 1 Section of Astrophysics, Astronomy and Mechanics, University of Athens, Panepistimiopolis, 157-84, Athens, Hellas, Greece \\ e-mail: obitzar@cc.uoa.gr; e-mail: elivan@cc.uoa.gr \\ ${ }^{2}$ Institute of Astronomy, The Observatories, Madingley Road, Cambridge, CB3 OHA, UK \\ e-mail: cat@ast.cam.ac.uk \\ 3 Astronomical Institute “Anton Pannekoek”, University of Amsterdam \& Centre for High Energy Astrophysics, Kruislaan 403, \\ 1098 SJ Amsterdam, The Netherlands \\ e-mail: edvdh@astro.uva.nl
}

Received 12 August 2002 / Accepted 7 October 2003

\begin{abstract}
We discuss possible evolution channels that lead to the formation of luminous supersoft X-ray sources, subclasses of which may be progenitors of type Ia supernovae. We carry out full evolution calculations from the zero-age main sequence to the supersoft source. A novel feature of our calculations is the inclusion of thermohaline mixing after mass transfer during binary evolution. The main effect of this is to produce secondaries of non-solar composition. Candidate initial progenitors are intermediate-mass donors of about $7 M_{\odot}$ with companions in the range 1.5-3.0 $M_{\odot}$. We concentrate on early case-C evolution, which means that the primary fills its Roche lobe when it ascends the Early Asymptotic Giant Branch while its core is highly evolved and massive enough to form a CO white dwarf. A crucial role, established by observations in this part of HR diagram, is played by mass loss in winds and we treat winds with a new approach. Since common-envelope evolution (CE) is generally invoked to explain the formation of close binaries with one or two degenerate components, we assume that the progenitors undergo severe mass and angular momentum loss through such a phase. We further study how the configurations of the postCE systems, composed of a massive white dwarf and a 1.5-3.0 $M_{\odot}$ companion, depend on the parameters of CE-evolution and mass-loss rates in various phases of evolution. Under these general assumptions a new path for the formation of SSSs is found which differs from that of the, usually assumed, solar composition donors. Our results may explain supersoft systems with enhanced helium abundances such as U Sco and very luminous extragalactic supersoft sources such as CAL 83 in the LMC and possibly the CHANDRA source (N1) in M 81.
\end{abstract}

Key words. X-rays: binaries - stars: formation, evolution

\section{Introduction}

Supersoft X-ray Sources (hereinafter SSSs) have been explained with the following model (van den Heuvel et al. 1992; Southwell et al. 1996) supported by observations (Greiner 2002, 2000, 1996; Swartz et al. 2002). A companion star transfers mass on a thermal time scale $\left(10^{6}-10^{7} \mathrm{yr}\right)$ via Roche lobe overflow to its less massive white dwarf companion. The accreted hydrogen-rich material burns steadily at the white dwarf surface at a high rate of the order of $10^{-7} M_{\odot} \mathrm{yr}^{-1}$. This model (hereinafter referred as WD+MS system) fits well with the X-ray and optical properties of SSSs (e.g. CAL 87) if the companion has a mass of 1.3-2.5 $M_{\odot}$ (Kahabka \& van den Heuvel 1997).

Here we investigate a new pathway for the formation of SSSs from WD+MS systems in which the hydrogenburning donor has a non-solar composition owing to thermohaline mixing of $\mathrm{He}$ rich material into its radiative envelope.

Send offprint requests to: O. M. Bitzaraki, e-mail: obitzar@cc.uoa.gr
This evolutionary path, including the mass and angular momentum loss driven by winds, we describe as evolution with thermohaline-mixing or case $2 \mathrm{a}$ distinguished from case $2 \mathrm{~b}$ which we reserve for conventional WD+MS systems. The enhanced winds included in our evolution code allow us to fit the newly revised initial to final mass relation of white dwarfs (Weidemann 2000). The main consequence of their implementation is to keep the final WD core mass of a $7 M_{\odot}$ star at about $1 M_{\odot}$. Without them the $\mathrm{CO}$ core mass reaches about $1.4 M_{\odot}$ which is inconsistent with observations. When the final core mass exceeds the Chandrasekhar mass we call it case 1 . This leads to the formation of a NS. Here we concentrate on case 2 because we are interested in the formation of WDs.

We calculate the evolution of the primary through commonenvelope and post-CE phases up to the formation of a $\mathrm{CO}$ white dwarf. Our evolutionary computations describe two scenarios for the formation of a WD+MS system, a) the non-solarlike MS donors and $\mathrm{b}$ ) the conventional scenario of solarlike donors in candidate SSSs. Subsequently we evolve the WD+MS systems produced and draw conclusions about their final fate. In Sect. 2 
we give an account of the input parameters to the evolution code that we employed for the whole set of computations. In Sect. 3.1.1 we describe the way the mass loss by stellar winds from the primary is calculated. In Sects. 3.1.2 and 3.1.3 CE calculations are discussed together with our criterion for their termination. In Sect. 3.1.4 we present the calculation of post CE evolution. In Sect. 3.1.5 we define the two cases of evolution that are computed in the present work. In Sect. 4, we give the results of our computations for both cases of evolution. In Sects. 5 and 6 we discuss in detail the evolution of the primary and the secondary for case $2 \mathrm{a}$ and $2 \mathrm{~b}$ of evolution, respectively. In Sect. 7 we give our conclusions for CE evolution. In Sect. 8, we compare our results with observed data and finally, in Sect. 9, we outline the major conclusions of this study.

\section{Basic assumptions}

The latest version of the Eggleton code (Eggleton 1971, 1972, 1973b; Eggleton et al. 1973a) as updated by a number of authors (see below) was employed to carry out the calculations. An important feature of the code is the use of a self-adaptive, non-Lagrangian mesh spacing function. The treatment of convection, semiconvection and overshooting is based on a diffusion process. The code solves simultaneously the equations for the structure and the diffusion equations for the chemical composition. The non-Lagrangian mesh-spacing function depends on the local pressure, temperature, Lagrangian mass and radius. A stellar evolution track can be computed quite accurately with only 200 mesh points up to core helium flash, for stellar masses less than $2.5 M_{\odot}$, and up to central carbon burning, for masses less than 7-8 $M_{\odot}$. Improvements to the code made by Pols et al. (1995) include pressure ionisation and Coulomb interactions in the equation of state and new opacity tables of Rogers \& Iglesias (1992, OPAL) and Alexander \& Ferguson (1994a, 1994b). Nuclear reaction rates according to Caughlan et al. (1985) and Caughlan \& Fowler (1988) are used, while neutrino loss rates are taken from Itoh et al. (1989, 1992, 1996). Some changes to the code are also described by Han et al. (1994). A bicubic spline interpolation in opacities at low temperatures was introduced by Tout et al. (1996). Details of the treatment of the convective overshooting are given in Pols et al. (1998). More recent improvements in the code focus on the mesh-spacing function and precise atomic masses of the chemical species were introduced by Pols \& Tout (2000).

\subsection{General assumptions for the primary star}

Throughout the evolution we use primary to mean the star which is initially the more massive component. We consider systems in which it has a mass around $7 M_{\odot}$, close to the lower limit for core-collapse. This mass is representative for the mass range $\left(4-7 M_{\odot}\right)$ that produces massive CO WDs. We evolve the star from the zero-age main sequence up to the end of the early asymptotic giant branch terminated by the second dredge-up. General assumptions for the evolution of primaries are listed below.
1. The initial chemical composition of the primary is solar $(X=0.70, Y=0.28, Z=0.02)$.

2. A mixing length parameter of $\alpha=2.0$ is used. Convective overshooting is not allowed.

3. Mass loss in winds is as described in Sect. 3.1.1.

4. The donor transfers mass via Roche lobe overflow during EAGB evolution, when it has developed a small $\mathrm{CO}$ core (Case $\mathrm{C}$ mass transfer).

5. Common envelope evolution (CE) is modelled as in Sects. 3.1.2 and 3.1.3.

6. Post-CE evolution takes account of i) mass-loss through winds or Roche lobe overflow and ii) angular momentum losses driven by radiative winds, gravitational radiation or magnetic braking (Sect. 3.1.4).

\subsection{General assumptions for the evolution of the secondary star}

We use secondary to mean the star which is initially the less massive component. The evolution of secondary stars of $1.5,1.7,2.0,2.5,3.0 M_{\odot}$ is computed until the secondary star becomes a helium white dwarf, if less massive than $2 M_{\odot}$, and carbon ignition if more massive. This mass range enables the MS secondary to fill its Roche lobe as a consequence of radius expansion driven by the nuclear evolution. Main assumptions for these computations are:

1. The initial chemical composition of the secondary is solar ( $X=0.70, Y=0.28, Z=0.02$ ). However, there are some cases in which the secondary is of non-solar composition under specific circumstances explained in Sect. 3.1.5.

2. The mixing length parameter is $\alpha=2.0$. Convective overshooting is included in accordance with observations (Pols et al. 1998).

3. Mass loss in winds is not included because we are mainly interested in the evolution of these stars during their mainsequence and Hertzsprung-gap lives, when such mass loss is small.

4. Common envelope evolution may occur for these systems if the secondary overfills its Roche lobe under conditions examined in Sect. 3.1.3.

5. Accretion on to a WD (or a neutron star) from the secondary's wind is negligible, both because of the cross section of the accretor and the low radiation-driven mass-loss rates induced from less-massive stars $\left(M \leq 3.0 M_{\odot}\right)$. The evolution is driven by i) mass loss through Roche lobe overflow or ii) angular momentum loss through magnetic braking or the emission of gravitational waves.

\section{Description of the model}

We describe how we implement the relatively uncertain physics of stellar winds and common-envelope evolution. 


\subsection{Primary star}

\subsubsection{Mass-loss by stellar winds from the primary}

We implement mass loss according to the formulae described by Hurley et al. (2000). For all stars with radiative envelopes mass loss is as prescribed by de Jager et al. (1988), Nieuwenhuijzen \& de Jager (1990) and Kudritzki et al. (1989):

$$
\begin{aligned}
\dot{M}_{\mathrm{NJ}}= & 9.6 \times 10^{-15}\left(\frac{Z}{Z_{\odot}}\right)^{\frac{1}{2}}\left(\frac{R}{R_{\odot}}\right)^{0.81} \\
& \times\left(\frac{L}{L_{\odot}}\right)^{1.24}\left(\frac{M}{M_{\odot}}\right)^{0.16} M_{\odot} \mathrm{yr}^{-1} .
\end{aligned}
$$

For red giants and beyond, Reimers' mass loss (Kudritzki \& Reimers 1978) is applied according to

$\dot{M}_{\mathrm{R}}=4 \eta \times 10^{-13} \frac{\left(L / L_{\odot}\right)\left(R / R_{\odot}\right)}{M / M_{\odot}} M_{\odot} \mathrm{yr}^{-1}$,

with $\eta=0.4 \pm 0.2$ to fit the horizontal branch morphology in Globular Clusters (Iben \& Renzini 1983; Carraro 1996).

When a star ascends the thermally pulsing AGB (TPAGB), mass loss increases and depends on the pulsation period $P_{0}$ (Vassiliadis \& Wood 1993):

$$
\begin{aligned}
\log \left(\frac{\dot{M}_{\mathrm{VW}}}{M_{\odot} \mathrm{yr}^{-1}}\right)= & -11.4+0.0124\left(\frac{P_{0}}{d}\right) \\
& -100 \max \left\{\left(\frac{M}{M_{\odot}}-2.5,0\right)\right\},
\end{aligned}
$$

where

$\log \left(\frac{P_{0}}{\mathrm{~d}}\right)=-2.07-0.9 \log \frac{M}{M_{\odot}}+1.94 \log \frac{R}{R_{\odot}}$.

Before any superwind, $\dot{M}$ is limited to a maximum of $\dot{M}_{\mathrm{VW}, \max }=1.36 \times 10^{-9} M_{\odot} \mathrm{yr}^{-1}$.

New data on the white dwarfs in the Hyades, NGC 3532 and NGC 2516 allowed the revision of the initial to final mass relation for masses up to $7 M_{\odot}$ (Weidemann 2000). This restricts the upper mass limit for $\mathrm{CO}$ white dwarfs to $1.0 \mathrm{M}_{\odot}$ and this limits the core mass at the onset of thermal pulses on the AGB.

In order to obtain a final mass of the $\mathrm{CO}$ core of the order of $1.0 M_{\odot}$ from a $7.0 M_{\odot}$ progenitor there is a need to introduce superwinds of the order of $10^{-4} M_{\odot} \mathrm{yr}^{-1}$, so that the whole hydrogen-rich envelope of a single star can be removed on the AGB before the core mass reaches the Chandrasekhar limit. These high mass-loss rates are expected to produce circumstellar shells around AGB stars as already observed in Mira and OH/IR stars (Knapp \& Morris 1985). The method applied to calculate the superwind is described in detail by Blöcker (1995). It sets in at pulsation periods longer than about $100 \mathrm{~d}$. Reimers' rate is suitably modified to describe smoothly the transition to Bowen-like superwinds (Bowen 1988) with a rate $\dot{M}_{\mathrm{B}}$. Formulae for these have been constructed with dynamical modelling of long-period variable-star atmospheres for a grid of Mira-like stars with masses ranging from 0.8-2.0 $\mathrm{M}_{\odot}$ and fundamental mode pulsation periods from 175 to $1000 \mathrm{~d}$. Longer period models resemble OH/IR sources with optically thick circumstellar dust and $\dot{M}>10^{-5} M_{\odot} \mathrm{yr}^{-1}$, while shorter period stars resemble Mira variables with less dust and $\dot{M} \leq$ $10^{-6} M_{\odot} \mathrm{yr}^{-1}$ (Bowen 1988). The transition point is taken to be at a pulsation period $P_{0}=100 \mathrm{~d}$ which corresponds to a luminosity given by

$\log \frac{L_{\mathrm{tr}}}{L_{\odot}}=3.08+0.78 \log \frac{M}{M_{\odot}}$.

Below this $\dot{M}=\dot{M}_{R}$ and above it $\dot{M}=\dot{M}_{B}$. The relation between these rates is

$\dot{M}_{\mathrm{B}}=\Gamma(M)\left(\frac{L}{L_{\odot}}\right)^{2.7} \dot{M}_{R}$,

where

$\Gamma(M)=4.83 \times 10^{-9}\left(\frac{M}{M_{\odot}}\right)^{-2.1}$.

So

$\dot{M}_{\mathrm{B}}=4.83 \times 10^{-9}\left(\frac{M}{M_{\odot}}\right)^{-2.1}\left(\frac{L}{L_{\odot}}\right)^{2.7} \dot{M}_{R}$.

With Eq. (8) the evolution on the AGB is described well, so we use it in all evolutionary calculations with an upper limit $\dot{M}_{\mathcal{M}} \approx 5 \times 10^{-4} M_{\odot} \mathrm{yr}^{-1}$ in agreement with observations (Knapp $\&$ Morris 1985). During the superwind phase the remaining envelope of a single $7-M_{\odot}$ star is blown away to leave a core mass of $1 M_{\odot}$.

When an evolved star depletes its hydrogen envelope it develops a Wolf-Rayet-like mass loss described by Hamann et al. (1995) and Hamann \& Koesterke (1998) and suitably reduced by Hurley et al. (2000) to

$\dot{M}_{\mathrm{WR}}=10^{-13}\left(\frac{L}{L_{\odot}}\right)^{1.5}(1.0-\mu) M_{\odot} \mathrm{yr}^{-1}$,

where

$\mu=\left(\frac{M-M_{\mathrm{c}}}{M}\right) \min \left\{5.0, \max \left[1.2,\left(\frac{L}{L_{0}}\right)^{\kappa}\right]\right\}$,

$M_{\mathrm{c}}$ is the core mass defined as the hydrogen deficient region of the star and $\kappa$ is a constant introduced to enable the star to move across the H-R diagram towards the start of the WD cooling curve when the envelope mass becomes very small (Hurley et al. 2000). Here $L_{0}=7.0 \times 10^{4} L_{\odot}$ and $\kappa=-0.5$ for normal giants. Finally, for very luminous stars beyond the HumphreysDavidson (1994) limit, we add LBV-like mass loss,

$$
\begin{aligned}
\dot{M}_{\mathrm{LBV}}= & 0.1\left(10^{-5} \frac{R}{R_{\odot}}\left(\frac{L}{L_{\odot}}\right)^{1 / 2}-1.0\right)^{3} \\
& \times\left(\frac{L}{6 \times 10^{5} L_{\odot}}-1.0\right) M_{\odot} \mathrm{yr}^{-1} .
\end{aligned}
$$

For naked helium stars $(\mu=0)$ we have:

$\dot{M}=\dot{M}_{\mathrm{WR}}(\mu=0) M_{\odot} \mathrm{yr}^{-1}$.

As the white dwarf cools it experiences a few shell flashes on its surface. These do not affect the evolution but are numerically 
awkward so we avoid them by increasing the mass loss rate to $10^{4}$ times Reimers' rate when the luminosity $L>10^{4.2} L_{\odot}$. Enhanced mass-loss rates for the cases of post-CE evolution when super-Eddington luminosities would be encountered are applied. This allows us to evolve the cooling of the post-CE remnant to the WD branch. We use that given by Tout \& Hall (1991) which enhances a standard Reimers' rate by a factor of up to $10^{4}$.

\subsubsection{Common envelope evolution}

In general when the mass ratio $q=M_{1} / M_{2}$ of a binary system exceeds 0.8 or so at the moment the primary fills its Roche lobe as a giant the system is expected to experience common envelope evolution. When the donor (initially the primary) has developed a small CO core on the EAGB and fills its Roche lobe it starts transferring mass (Case-C mass transfer) to its companion. A donor which, in a wide binary, has developed a deep convective envelope before Roche lobe overflow and is still more massive than its companion begins transferring mass unstably because the convective envelope of the giant responds to mass loss with a rapid expansion while the orbit, and hence the Roche lobe, shrinks. As a result of mass transfer from the more to the less massive star the mass-loss rate increases. Under these conditions, the secondary cannot attain thermal equilibrium because the new layer is accreted on a timescale approaching the dynamical timescale of the massive donor. This is much shorter than the thermal timescale of the secondary star. As a result, the added matter expands to fill the Roche lobe of the secondary. An extended circumbinary envelope engulfs both components. Drag forces between the two stellar cores and the common envelope cause the cores to spiral in towards each other. During this process some of the orbital energy is transformed into kinetic energy in the envelope, expelling it from the system into interstellar space. When most of the envelope has been lost, a compact system is left as the nucleus of a planetary nebula with a close binary companion. Whether or not the CE phase occurs depends on the mass ratio $q$, the extent of the convective envelope of the donor star and the evolutionary state of the accretor. The more evolved the donor is, the greater is the discrepancy between the thermal readjustment timescale of the accretor and the mass-transfer timescale and the more likely it is that a CE phase takes place (Iben \& Livio 1993). These conditions are fulfilled in the systems we evolve.

Although our treatment of the CE is simple, a 3D hydrodynamical approach would be beyond the purpose of this paper. Nevertheless, CE is considered in a more detailed way than in some previously published papers (Dewi \& Tauris 2000). The post-CE and spiral-in phase parameters of the problem are estimated by means of Webbink's formula (Webbink 1984) which is based on energy arguments. The hydrodynamic work of Taam and associates (e.g. Taam 1996; Taam \& Sandquist 2000) shows that such an approach is justified. Here $M_{\mathrm{d}}$ and $M_{2}$ are the masses of the donor star and its companion and $a_{\mathrm{f}}$ and $a_{\mathrm{i}}$ represent the post $\mathrm{CE}$ and initial orbital separation. Let us denote by $\alpha_{\mathrm{CE}}$ the efficiency for the conversion of orbital energy $\delta E_{\text {orb }}$ into kinetic energy of the envelope expulsion and $R_{\mathrm{L}} \approx R_{\mathrm{d}}$ the Roche lobe radius and donor's radius. Then Webbink's formula gives an estimate for the post-CE separation if the entire envelope is lost during the $\mathrm{CE}$ at the expense of orbital binding energy:

$\delta E_{\text {orb }}=-\frac{G M_{\mathrm{c}} M_{2}}{2 a_{\mathrm{f}}}+\frac{G M_{\mathrm{d}} M_{2}}{2 a_{\mathrm{i}}}$,

where $M_{\mathrm{c}}$ is the core mass of the primary and

$\alpha_{\mathrm{CE}}=\frac{E_{\mathrm{env}}}{\delta E_{\mathrm{orb}}}$.

The binding energy $E_{\mathrm{env}}$ of the envelope to the core is calculated with de Kool's (1990) formula:

$E_{\mathrm{env}}=-\frac{G M_{\mathrm{d}} M_{\mathrm{env}}}{\lambda a_{\mathrm{i}} R_{\mathrm{L}}}$

where $M_{\text {env }}$ is the envelope mass $\left(M_{\mathrm{d}}=M_{\mathrm{c}}+M_{\mathrm{env}}\right)$. The parameter $\lambda$ describes the total binding energy of the envelope. It depends on both the kinetic (thermal) energy and the density distribution of the star and we calculate how it varies throughout the evolution (typically 0.5 to 4.0) instead of assuming a constant value of 0.5 (de Kool 1990). Combining the above equations we find

$\frac{a_{\mathrm{f}}}{a_{\mathrm{i}}}=\frac{M_{\mathrm{c}} M_{2}}{M_{\mathrm{d}}} \frac{1}{M_{2}+2 M_{\mathrm{env}} /\left(\alpha_{\mathrm{CE}} \lambda R_{\mathrm{L}}\right)}$.

In calculating $\lambda$ we use the virial theorem. This is a good approximation for the EAGB when the envelope is composed of ionised hydrogen and helium and recombination processes do not contribute significantly (Dewi et al. 2000). Then

$E_{\text {bind }}=-\int_{M_{\mathrm{c}}}^{M_{\mathrm{d}}} \frac{1}{2} \frac{G m}{r} \mathrm{~d} m$,

where $r$ is the radius enclosing mass $m$.

Hydrodynamical calculations show that in a real binary system not all of the envelope is lost. Rather some remains around the core at the end of CE. We simulate this by calculating the binary evolution as we gradually strip the donor with an artificial wind. Important assumptions are that $M_{\mathrm{c}}$ remains unchanged during the ejection process and that no exchange of energy occurs between core and envelope. Systems survive CommonEnvelope and spiral-in phase if the secondary star does not fill its Roche lobe at the termination of this stage.

\subsubsection{Simulation of the CE evolution}

We follow step by step the change of the orbital elements during an artificial CE stage by stripping the donor gradually. Though the precise evolution of a star during CE evolution and the associated spiral-in is still undetermined, partly because it occurs on a dynamical time scale, we treat it in the following way:

a) We use a star with the same structure as that of donor when it fills its Roche lobe to model the CE.

b) This star is further assumed not to evolve nuclearly because of the short CE timescale. 
c) The mass loss during the CE phase is represented by an artificial constant-rate wind with $10^{-3}<\dot{M}_{\mathrm{w}} / M_{\odot} \mathrm{yr}^{-1}<10^{-4}$. Note that much higher mass-loss rates for $\mathrm{CE}$ of the order $10^{-1} M_{\odot} \mathrm{yr}^{-1}$ are expected (Regös \& Tout 1995; Taam $\&$ Sandquist 2000) but after a set of numerical experiments, we found that $\dot{M}_{\mathrm{w}}=M_{\mathrm{d}} / t_{\mathrm{KH}}$ does not disturb the physics of the CE. For much higher mass-loss rates a 3D hydrodynamical approach would be required. It should be stressed that this wind is purely artificial to calculate how, with Eq. (17), the orbit changes as the envelope mass decreases.

d) We follow the change of the orbital separation with Webbink's formula. At every time-step the mass lost $\delta M$ from the donor is

$\delta M=\left|\dot{M}_{\mathrm{w}}\right| \delta t$

and the corresponding binding energy of the envelope mass that is carried off is

$\delta E_{\mathrm{bind}}=\frac{\delta M}{M_{\mathrm{env}}} E_{\mathrm{bind}, 0}$,

where $E_{\text {bind, } 0}$ is the binding energy of the envelope at the start of the CE phase.

e) The change of the orbital separation is calculated from Eqs. (16), (17), (19) and (20),

$\frac{a_{\mathrm{t}+\mathrm{dt}}}{a_{\mathrm{t}}}=\frac{M_{\mathrm{d}, \mathrm{t}+\mathrm{dt}}}{M_{\mathrm{d}, \mathrm{t}}}\left(1+\frac{2\left|\delta E_{\mathrm{bind}}\right| a_{\mathrm{t}}}{G M_{\mathrm{d}, \mathrm{t}} M_{2} \alpha_{\mathrm{CE}}}\right)^{-1}$,

where $M_{\mathrm{d}, \mathrm{t}+\mathrm{dt}}$ and $M_{\mathrm{d}, \mathrm{t}}$ are the final and initial donor masses at each time step, with $M_{\mathrm{c}}=M_{\mathrm{d}, \mathrm{t}+\mathrm{dt}}$ and $M_{\mathrm{d}}=M_{\mathrm{d}, \mathrm{t}}$, and $E_{\text {env }}=\delta E_{\text {bind }}$. The companion mass $M_{2}$ remains unchanged. We select $\alpha_{\mathrm{CE}}$ so that the star does not fill its Roche lobe at the end of the CE phase. For this purpose we constructed a set of figures such as Fig. 9. This depicts final orbital radii versus initial orbital separation at the onset of the CE phase and similarly final orbital periods as a function of initial orbital periods when $\mathrm{CE}$ ensues. The final orbital separation at the end of the CE phase is compared to the critical separation at which the companion star (the secondary) fills its Roche lobe $\left(a_{\mathrm{f}} \geq a_{\mathrm{cr}}\right.$ or $P_{\mathrm{f}} \geq P_{\mathrm{cr}}$ ). The minimum value of $\alpha_{\mathrm{CE}}$ that satisfies this condition was employed for the surviving systems.

f) We assume no mass-accretion on to the secondary component so the Roche lobe radius can be calculated according to Eggleton's formula (1983),

$R_{L}=\frac{0.49 a}{0.6+q^{-2 / 3} \ln \left(1+q^{1 / 3}\right)}$

where $q=M_{\mathrm{d}} / M_{2}$ is the mass ratio and $a$ is the orbital separation.

g) Finally, CE evolution is stopped when the donor underfills its Roche lobe, $R_{\mathrm{d}} \leq R_{\mathrm{L}}$. This is a significant assumption because it sets the physical condition for the termination of the CE and spiral-in. In these evolutionary models this assumption is satisfied in a region between the lower boundary of the convective envelope and the hydrogen burning shell. Under these conditions, there is no physical reason to set the core mass of the massive donor inside the helium burning shell, because, the star has already started shrinking inside its Roche lobe earlier in spiral-in when mass just above the hydrogen-shell burning was being expelled. The shrinkage of the donor inside its Roche lobe is a consequence of the response of the radiative envelope of the donor to mass loss. Were this region to be convective the response of the envelope would be a rapid expansion beyond the limiting surface of Roche lobe radius (Soberman et al. 1997). This definition of the boundary of the core inside the hydrogen-shell burning is justified in a natural way.

\subsubsection{Post CE-Evolution}

We calculate post-CE evolution of the remnants (subdwarfs) by assuming a) loss of mass and angular momentum are driven by winds and $b$ ) angular momentum loss by gravitational radiation or magnetic braking. The angular momentum loss $\dot{J}_{\text {orb }}$ owing to gravitational radiation is given by (Landau \& Lifshitz 1965):

$\frac{\dot{J}_{\text {orb }}}{J_{\text {orb }}}=-\frac{32 G^{3}}{5 c^{5}} \frac{M_{1} M_{2}\left(M_{1}+M_{2}\right)}{a^{4}}$,

where $M_{1}$ and $M_{2}$ are the two masses orbiting one another at a distance $a, c$ is the speed of light and $J_{\text {orb }}$ is the angular momentum of the binary,

$J_{\text {orb }}=M_{1} M_{2} \sqrt{\frac{G a}{M_{1} M_{2}}}$.

For the hot post-CE remnants this is the important mechanism. However, later in the evolution, when the secondary transfers mass to the WD, magnetic braking may become important. The magnetic field of the donor forces the wind matter to corotate out to many stellar radii. Any loss of angular momentum caused by the spin-down of the donor star is transferred to the orbit by tidal forces. A formula based on semi-empirical laws for single G stars (Rappaport et al. 1983) is

$\frac{\dot{J}_{\mathrm{mb}}}{J_{\text {orb }}}=\frac{-3.8 \times 10^{-30} R_{\odot}^{4-\gamma}\left(M_{1}+M_{2}\right) R_{2}^{\gamma} \omega^{2}}{M_{1} a^{2}} \mathrm{~s}^{-1}$,

where $\omega$ is the angular velocity of the donor and $\gamma$ a parameter that determines the dependence of the braking on the radius of the star. We have set $\gamma=2$ because this fits well with observations.

\subsubsection{Cases of binary evolution and their development after CE-phase}

We define case 1 as the classical binary evolution with no winds from ZAMS to TPAGB in order to distinguish it from case 2 when winds are included. If winds are suitably taken into account then a smaller final core mass at the end of CE phase results. Post-CE remnants with core masses $M_{1 \mathrm{~F}}$ less than $1.4 M_{\odot}$ $\left(M_{1 \mathrm{~F}} \leq 1.4 M_{\odot}\right)$ evolve to form WDs. From a $7 M_{\odot}$ star a CO WD with mass about $1 M_{\odot}$ is formed. We examine two evolutionary subcases for the post-CE remnant of the primary dependent on whether the remnant can or cannot again fill its Roche lobe during He-shell burning. The action of radiative pressure at Super-Eddington luminosities assisted with the enhanced tidal effects triggered by the increased proximity of the 
binary components could in turn amplify radiative winds. We consider whether these tidally enhanced radiative winds can drive the evolution 1) after the remnant star has filled its Roche lobe and reached a critical maximum luminosity beyond which no cooling of the WD would be possible. We call this possibility case $2 \mathrm{a}$. And 2) at the onset of Roche lobe overflow. If these winds are strong enough to prevent the remnant filling its Roche lobe, it starts shrinking inside its critical innermost equipotential surface. We refer to this case as case $2 b$ of evolution.

In case $2 \mathrm{a}$, the companion which is a MS star, despite accretion of He-rich material, manages to avoid expansion to a contact binary. This can be attained if, after accretion, some mechanism can efficiently mix the newly added He-rich material into the radiative envelope of the secondary on a timescale shorter than the thermal timescale. Such thermohaline mixing produces an homogeneous secondary. An initial $1.5 M_{\odot}$ secondary of solar composition that accretes $0.6 M_{\odot}$ He-rich material from the post-CE remnant of the primary is homogenised to $X=0.50$ and $Y=0.48$. The secondary then has a H-rich Heenhanced envelope in radiative equilibrium. We have found that the maximum mass that could be transferred through Roche lobe overflow is about $0.6 M_{\odot}$ increasing the secondary mass to $2.1 M_{\odot}$. Case 2 a therefore leads to non-solarlike chemical compositions.

In case $2 b$, the secondary star avoids accretion and maintains a solar-like composition because the enhanced winds keep the companion underfilling its Roche lobe. The former is a new evolution channel for SSSs while the latter could lead to conventional WD+MS systems.

\subsection{Secondary star}

When the secondary star overflows its Roche lobe it transfers mass to its now cool C/O-WD companion of about $1 M_{\odot}$. The mass and chemical composition of the secondary remain unchanged for case- $2 \mathrm{~b}$ evolution while for case $2 \mathrm{a}$ the mass increases by $0.6 M_{\odot}$ and it acquires a non-solar chemical composition. The orbital period is still that at the end of the CE phase. Among these systems those in which mass transfer rates can support steady nuclear burning on the WD surface are candidate SSSs which are possible progenitors of SNe Ia (Branch et al. 1995). We must keep track of the mass that is accreted on to the WD surface. This is a function of the mass-transfer rate and the WD mass and its temperature. To avoid the accreting WD swelling up to a giant we must invoke a stabilising wind as has Hachisu et al. (1996) and so we make the following assumptions:

$a_{1}$ ) For mass transfer rates in excess of a critical value

$\dot{M}_{\mathrm{cr}, \mathrm{H}}=8.5 \times 10^{-7}\left(\frac{M_{\mathrm{WD}}}{M_{\odot}}-0.52\right) M_{\odot} \mathrm{yr}^{-1}$

a strong disc wind (accretion wind) stabilises the mass transfer rate even if the mass-losing star has a deep convective envelope. We define the accumulation efficiency parameter of hydrogen $\alpha_{\mathrm{H}}=\dot{M}_{\mathrm{cr}} / \dot{M}$, where $\dot{M}$ is the mass transfer rate and $\Delta M=\alpha_{\mathrm{H}} \dot{M}_{\mathrm{cr}} \delta t$ is accreted at a constant rate $\dot{M}_{\mathrm{cr}}$ in time $\delta t$. The remaining matter $\left(\dot{M}-\dot{M}_{\mathrm{cr}}\right) \delta t$ blows off the interstellar medium.
Hydrogen burns steadily to helium and then on to carbon on the white dwarf surface. Its mass grows to $1.38 M_{\odot}$ when $\mathrm{C}$ ignites degenerately in the core and the star explodes as a type Ia supernova.

$a_{2}$ ) If the mass transfer rate is in the range $0.4 \dot{M}_{\text {cr }} \leq \dot{M} \leq$ $\dot{M}_{\text {cr }}$ (Nomoto 1982; Fujimoto 1982a, 1982b) steady nuclear burning of hydrogen can occur on the surface of the WD and none of the transferred mass is lost. Extensive grids of multicycle nova models calculated by Prialnik \& Kovetz (1995) provide us with information on the hydrogen accumulation efficiency parameter $\alpha_{\mathrm{H}}$ and its dependence on the mass-transfer rate, the mass and the temperature of the WD. We use the data given in Fig. 4 of Prialnik \& Kovetz (1995) for the case of cold white dwarfs with temperature $T_{\mathrm{WD}}=10^{7} \mathrm{~K}$. For $\dot{M} \leq 0.4 \dot{M}_{\mathrm{cr}}$ hydrogen burns unsteadily in flashes and part or all the envelope is lost.

$a_{3}$ ) If $3.0 \times 10^{-8} M_{\odot} \mathrm{yr}^{-1} \leq \dot{M} \leq 0.4 \dot{M}_{\text {cr }}$ weak $\mathrm{H}$ flashes occur on the surface of the white dwarf and $\alpha_{\mathrm{H}}$ is calculated according to Prialnik \& Kovetz (1995).

$\left.a_{4}\right)$ If $\dot{M} \leq 3.0 \times 10^{-8} M_{\odot} \mathrm{yr}^{-1}$ then a strong nova explosion occurs and the mass of the WD may decrease $\left(\alpha_{\mathrm{H}}=0.0\right.$ or, $\left.\alpha_{\mathrm{H}}<0.0\right)$ (Prialnik \& Kovetz 1995). We terminate our calculations in this case.

Throughout these calculations we assume hydrogen immediately burns to helium on the WD surface. This determines the helium accumulation rate. When the mass of He exceeds a minimum value of $\Delta M_{\mathrm{ig}}=4.8 \times 10^{-5} M_{\odot}$ for helium accretion rates $\dot{M}_{\mathrm{He}} \geq 1.7 \times 10^{-6} M_{\odot} \mathrm{yr}^{-1}$ (Kato \& Hachisu 1999), the helium shell burns at a rate equal to the hydrogen accumulation rate $\dot{M}_{\mathrm{He}}=\alpha_{\mathrm{H}} \dot{M}$.

$b_{1}$ ) According to Kato \& Hachisu (1999) if $-5.9 \leq$ $\log \left(\dot{M}_{\mathrm{He}} / M_{\odot} \mathrm{yr}^{-1}\right) \leq-5.0$ then the accumulation ratio $\alpha_{\mathrm{He}}=$ 1.0 , where $\alpha_{\mathrm{He}}$ is defined as the ratio of the processed matter remaining after one helium shell flash to that at ignition (Kato \& Hachisu 1999).

$b_{2}$ ) For helium accretion rates in the range $-7.4<$ $\log \left(\dot{M}_{\mathrm{He}} / M_{\odot} \mathrm{yr}^{-1}\right)<-5.9$, the mass accumulation efficiency parameter in helium shell flashes is $\alpha_{\mathrm{He}}=$ $-0.175\left(\log \dot{M}_{\mathrm{He}} / M_{\odot} \mathrm{yr}^{-1}+5.35\right)^{2}+1.05$ (Kato \& Hachisu 1999; Hachisu et al. 1999).

$\left.b_{3}\right)$ If $\log \left(\dot{M}_{\mathrm{He}} / M_{\odot} \mathrm{yr}^{-1}\right)<-7.4$ we set $\alpha_{\mathrm{He}}=0$. This means, that the strong helium flashes drive the expulsion of all processed matter. During this phase no mass is added to the white dwarf.

These new estimates of the accumulation ratio differ in some aspects from the previous results of Kato et al. (1989) because they use somewhat different opacity tables.

\section{Computational procedure}

We present results for the evolution of the case 2 binary system $7+1.5 M_{\odot}$ with an initial period of $350 \mathrm{~d}$ which leads to Case $\mathrm{C}$ evolution. The primary fills its Roche lobe at $t=4.845 \times 10^{7} \mathrm{yr}$ on the AGB by which time the convective envelope already extends to great depth, $R_{\text {conv }} / R_{\mathrm{d}}=0.89$. 


\subsection{Case 2a: Evolution with thermohaline mixing}

Case 2 a denotes the evolution of a donor that undergoes Roche lobe overflow during He shell burning without significantly enhanced winds. However at the end of Roche lobe overflow, when the luminosities are super-Eddington, it is possible that this wind drives the evolution of the post-CE remnant to a white dwarf. This is our case 2a. During mass transfer thermohaline mixing begins as the secondary accretes He-rich matter from the post-CE remnant and mixes all the transferred material through its outer radiative layers. In this way a non-solarlike secondary of greater mass is formed. We illustrate the detailed evolution of the primary in Fig. 1 (track A in the upper panel), 2 and 3 . The evolution of the secondary is described in Fig. 1 (track B in the upper panel) and 6.

\subsection{Case 2b: Evolution leading to conventional systems}

Case $2 \mathrm{~b}$ is the more classical evolution in which the companion (secondary) does not accrete during the primary's helium shell burning because we deliberately increase the mass-loss rate to $10^{4}$ times Reimers' before any mass is transferred. The secondary does not grow in mass nor does it change chemical composition. Again, whether or not the post-CE donor overflows its Roche lobe depends on the strength of the stellar wind from this star. At high rates it does not fill its Roche lobe again (Iben \& Livio 1993). This can be seen from the comparison of Fig. 2 (upper and lower) and Fig. 2 (upper), 4 which give the $\dot{M}$ evolution for both cases of evolution. Figure 1 (track A in the lower panel), 2(upper), 4 and 5 represent the evolution of the primary star while Fig. 1 (track B in the lower panel) and 7 the evolution of the secondary star.

\section{Detailed evolution of the primary component}

Figure 1 (upper panel) shows case 2 a evolution for both binary components in the HR diagram. This new subcase takes into account the effect of thermohaline mixing on the evolution of binary members. The location of the donor star at various evolutionary states is denoted by letters $\mathrm{A}_{i}$. At $\mathrm{A}_{1}$ it fills its Roche lobe and a CE ensues (dashed line). Its radius is then $R=167 R_{\odot}$. At $\mathrm{A}_{2}$ it first underfills its Roche lobe after a CE spiral-in phase of $3.7 \times 10^{4} \mathrm{yr}$. After a further time of order the thermal timescale of the envelope, when $R / R_{\mathrm{L}} \approx 0.85$ the $\mathrm{CE}$ phase comes to an end at point $\mathrm{A}_{3}$ about $4.0 \times 10^{4} \mathrm{yr}$ from its first Roche lobe overflow on the EAGB. It should be noted that these times are artificial because we have calculated the mass loss during the CE phase by introducing an artificial wind - see above. The real hydrodynamical timescale for CE evolution may be much shorter (Taam 1996; Taam \& Sandquist 2000). At $\mathrm{A}_{4}$ the donor has lost all of its H-rich envelope. Subsequently its remnant again fills its Roche lobe during $\mathrm{He}$ shell burning at $\mathrm{A}_{5}$. At $\mathrm{A}_{6}$ it detaches and contracts inside its Roche lobe once again. Up to $A_{5}$ cases $2 a$ and $2 b$ are the same. Points $A_{5}$ and $A_{6}$ are distinct for case $2 a$, while in case $2 b$, $\mathrm{A}_{5}$ coincides with point $\mathrm{A}_{6}$ because no Roche lobe overflow takes place. Figure 2 shows the mass-loss rate from the donor (solid line) from the ZAMS to the first Roche-lobe overflow on the EAGB, the CE, post-CE up to shortly after point $A_{6}$. The letters correspond to important phases as desribed in the table given. Figure 2 (lower panel) and 4 illustrate the difference between case $2 \mathrm{a}$ and case $2 \mathrm{~b}$ of evolution. As shown in Fig. 2 in case $2 \mathrm{a}$, a mass accretion phase of He-rich matter is allowed to the secondary if tidally enhanced winds are not important between points $A_{5}-A_{6}$. At $A_{6}$ the mass accretion is terminated by means of an artificial wind which relieves convergence problems caused by shell flashes on the surface of the star. This artificial wind was assumed to have the same formula as the tidally enhanced winds of case $2 b$ of evolution. We emphasize that this artificial wind has no effect on the long term evolution of the primary. The dot-dashed line gives the total donor mass and the dotted line the corresponding change of the $\mathrm{CO}$ core mass. Figure 3 presents the orbital separation (left axis) and orbital period (right axis). Tables 1 and 2 give numerical values at the points $\mathrm{A}_{i}, i=1, \ldots 8$.

In yet more detail, the primary first fills its Roche lobe on the EAGB at age $t=4.84 \times 10^{7} \mathrm{yr}$ and the CE phase begins. During this phase we adjust the mass-loss rate so that the donor continues to fill its Roche lobe, while diverging only marginally from thermal equilibrium, but does not evolve nuclearly. We terminate the CE phase when $R / R_{\mathrm{L}}<1$ by switching off the mass loss. We find that the post-CE remnant then attains thermal equilibrium when $R / R_{\mathrm{L}} \approx 0.8$. Two cases can be distinguished, those with non-degenerate $\mathrm{CO}$ cores and those that have developed degenerate cores before Roche lobe overflow. The model presented in Figs. 1-5 is the case of a nondegenerate core. The more evolved a system is the larger its radius and the smaller the binding energy of its envelope. This is illustrated in Fig. 8. Generally $\left|E_{\text {bind2 }}\right|>\left|E_{\text {bind1 }}\right|$, where index 1 refers to a core boundary outside and 2 inside the helium burning shell. An overall trend for the binding energy of the envelope to decrease with evolution is apparent from Fig. 8. So a more evolved star requires a smaller value of $\alpha_{\mathrm{CE}}$ because the envelope can be removed more easily. Models with non-degenerate cores require $\alpha_{\mathrm{CE}} \geq 3.0$ while for models with degenerate cores (Bitzaraki et al. 2003b) $\alpha_{\mathrm{CE}}$ can be as small as 1.0. Note further that if $M_{\mathrm{c}}=M_{\mathrm{H}}$ the release of orbital energy provides sufficient energy input to eject the envelope, while if $M_{\mathrm{c}}=M_{\mathrm{He}}$ other energy sources, such as tapping the stellar luminosity or ionisation energy, are required.

For a $1.5 M_{\odot}$ star with a radius of $1.5 R_{\odot}$ the final binary separation must be larger than $3.5 R_{\odot}$ so that the MS star does not fill its Roche lobe. For a donor star that fills its Roche lobe at the onset of the EAGB the final orbital separations, when $M_{\mathrm{c}}=M_{\mathrm{H}}$ are $0.92,1.84,3.67,5.51$ and $7.31 R_{\odot}$ for $\alpha_{\mathrm{CE}}$ of 0.5 , 1.0, 2.0, 3.0 and 4.0. The corresponding final orbital periods are $0.1,0.28,0.79,1.45$ and $2.24 \mathrm{~d}$. So for final orbital separations larger than $3.5 R_{\odot}$ we need $\alpha_{\mathrm{CE}}$ values greater than 2.0. However $\alpha_{\mathrm{CE}}=2.0$ corresponds to a situation where the secondary (being very close to ZAMS) fills its Roche lobe before the other star has evolved to a WD. So we require $\alpha_{\mathrm{CE}} \geq 3.0$ (see Fig. 9) if the system is to survive for an appreciable time after spiral-in. The final orbital separation as a function of the initial separation for the most general case where the star develops deep convective outer layers is presented in figures 

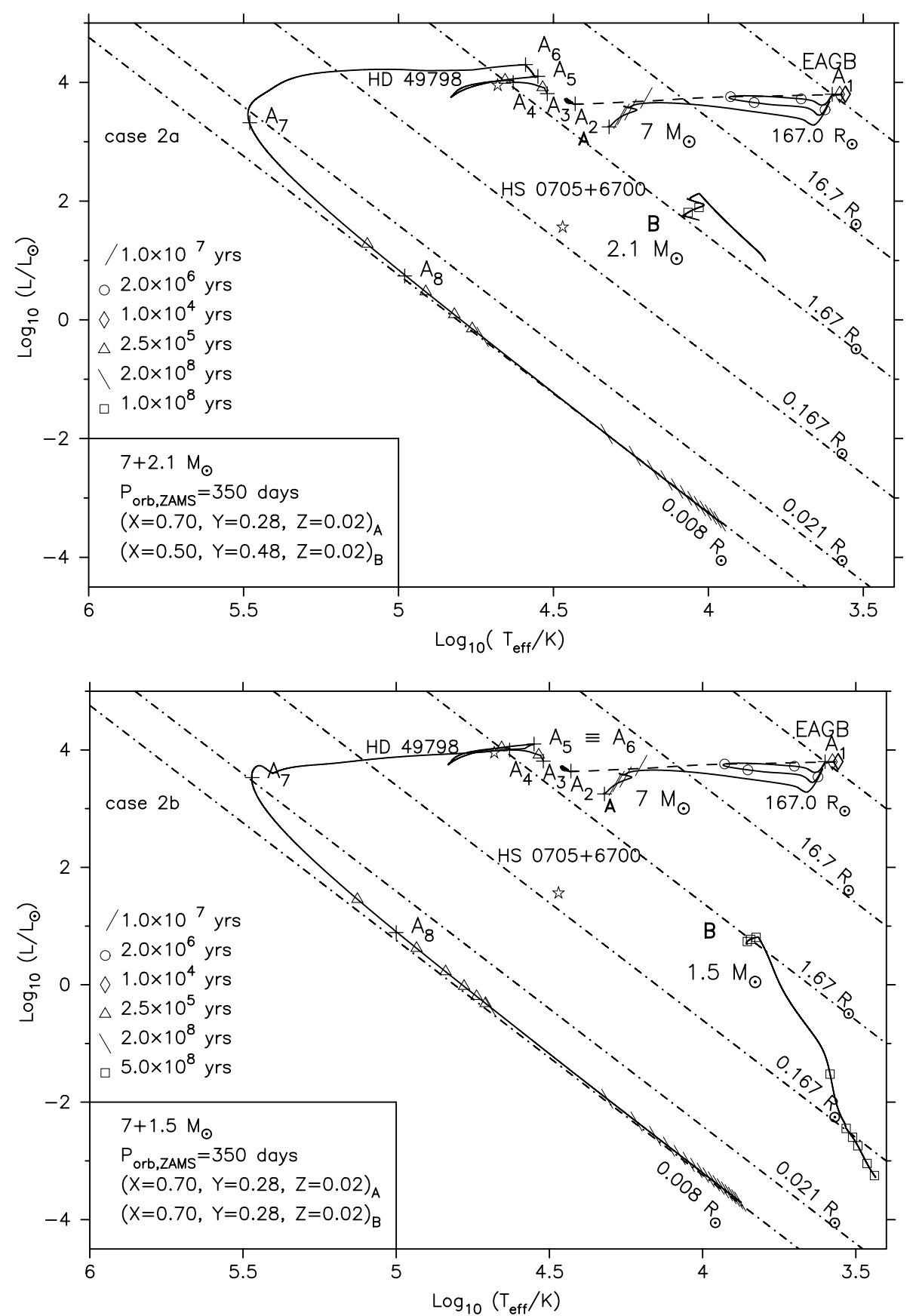

Fig. 1. Evolution in the H-R diagram of a system with initial component masses $7+1.5 M_{\odot}$ and initial orbital period (at ZAMS) of $350 \mathrm{~d}$. Case 2a (upper panel) has two phases of Roche lobe overflow while case $2 \mathrm{~b}$ (lower panel) has one. In case 2a, after the second Roche lobe overflow the secondary mass has reached $2.1 M_{\odot}$. In case $2 \mathrm{~b}$ the secondary does not accrete any mass because the post-CE remnant does not fill its Roche lobe again. Symbols, defined in the left-hand side of the figures, represent time intervals on the evolutionary tracks. The crosses correspond to the evolutionary phases $A_{1}$ through $A_{8}$ described in the text. Note the non-solar chemical composition of the secondary star owing to thermohaline-mixing.

similar to Fig. 10 for various values $\alpha_{\mathrm{CE}}$ when the core boundary is taken just inside the H-shell $\left(M_{\mathrm{c}}=M_{\mathrm{H}}\right)$ and He-shell $\left(M_{\mathrm{c}}=M_{\mathrm{He}}\right)$ burning.

In the model the final donor mass is $1.66 M_{\odot}$ consisting of a helium mantle of $1.08 M_{\odot}$ around a CO core of $0.58 M_{\odot}$. The smaller CO core mass at the end of the CE phase is a result of the cooling of the interior of the star owing to the lower gravitational energy production at increasingly smaller envelope masses. After the CE phase, the timescale of evolution is dictated by the rate of burning of the shell at the bottom of the H-rich envelope and by mass loss induced by winds. Our mass-loss rates are those of Hurley et al. (2000) as discussed in Sect. 3.1.1. After $4.2 \times 10^{4} \mathrm{yr}$ the star loses all its hydrogen-rich envelope and shrinks inside its Roche lobe. 


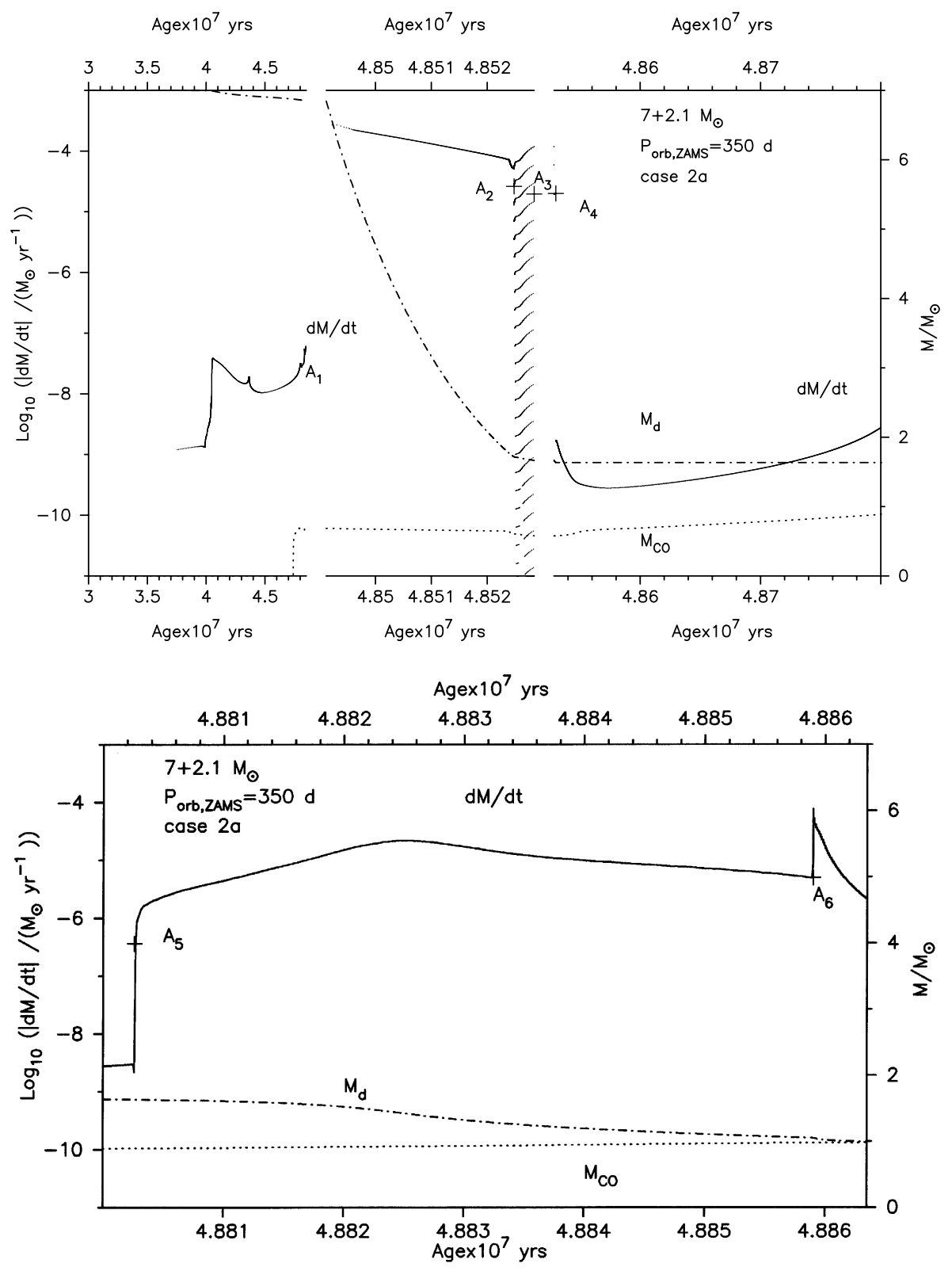

Fig. 2. Case $2 \mathrm{a}$ evolution of the primary for a system of initially $7+1.5 M_{\odot}$ stars and initial orbital period $350 \mathrm{~d}$ (see also Fig. 1). Upper panel: the time-variation of mass-loss rate $\dot{M}$ is indicated by the full line from ZAMS until $t=4.88 \times 10^{7} \mathrm{yr}$ (point $\mathrm{A}_{5}$ ). The evolution in this time interval is the same for both cases $2 \mathrm{a}$ and $2 \mathrm{~b}$ of evolution. The total mass of the primary versus time is denoted by the dot-dashed line while the mass of the $\mathrm{CO}$ core $M_{\mathrm{CO}}$ is shown with the dotted line. Points $\mathrm{A}_{1}-\mathrm{A}_{5}$ are defined in Table 1. Lower panel: Continued detailed diagram for the time evolution of $\dot{M}, M_{\mathrm{d}}$ and $M_{\mathrm{CO}}$ after $t=4.88 \times 10^{7} \mathrm{yr}$ for the time interval $\mathrm{A}_{5}-\mathrm{A}_{6}$. During this phase, He burns at a shell and if no enhanced winds play important role, He-rich mass is accreted to the secondary. As a result the secondary is enriched with helium under the thermohaline mixing effect. At a suitable time point $A_{6}$ a tidally enhanced wind as described in the text was assumed to overcome the surface shell flashes on the surface of the post-CE remnant of the primary which cause convergence problems to the code and allow the cooling of the star to the WD branch. This artificial wind does not change the long term evolution of the primary.

It drops from $2.46 R_{\odot}$ to $0.70 R_{\odot}$ when $L_{\mathrm{H}}=2.28 \times 10^{2} L_{\odot}$ and $L_{\mathrm{He}}=1.59 \times 10^{3} L_{\odot}$. Nuclear burning of He begins to be the dominant energy source in the subsequent evolution. After $3.1 \times 10^{5} \mathrm{yr}$ from the first Roche lobe overflow the donor fills its Roche lobe again as it evolves with a $\mathrm{He}$ burning shell (point $\mathrm{A}_{5}$ in the top $\mathrm{H}-\mathrm{R}$ diagram of Fig. 1). The donor is now $1.63 M_{\odot}$ with a degenerate $\mathrm{CO}$ core of $M_{\mathrm{CO}}=0.88 M_{\odot}$ and a radius of $3.0 R_{\odot}$. The mass transfer of He rich material begins on a nuclear timescale. At this point
$L=1.18 \times 10^{4} L_{\odot} \approx L_{\mathrm{He}}$ and $T_{\mathrm{eff}}=10^{4.55} \mathrm{~K}$. The surface chemical abundances are $X_{\mathrm{H}}=0.099, X_{\mathrm{He}}=0.88, X_{\mathrm{C}}=0.000$, $X_{\mathrm{N}}=0.013$ and $X_{\mathrm{O}}=0.0007$. The core is degenerate with $\log _{10}\left(\rho_{\mathrm{c}} / \mathrm{g} \mathrm{cm}^{-3}\right)=5.68$ and $\log _{10}\left(T_{\mathrm{c}} / \mathrm{K}\right)=8.58$.

In case $2 \mathrm{a}$ no extreme mass loss is encountered during helium-shell burning so this post-CE remnant fills its Roche lobe and begins transferring mass to its companion at the high rate of $10^{-6} M_{\odot} \mathrm{yr}^{-1}$ owing to expansion as processed matter is 


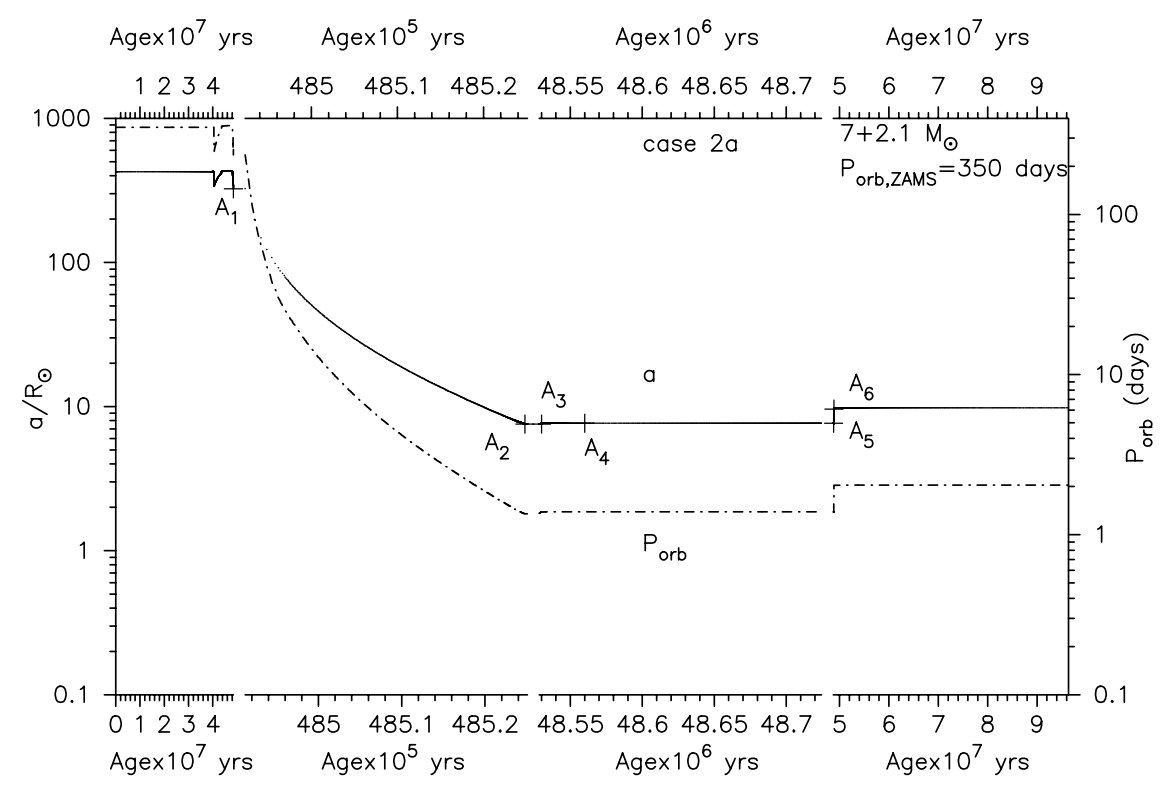

Fig. 3. Case 2 a evolution of the primary for a system of initially $7+1.5 M_{\odot}$, the time variation of the binary separation (full line) and the corresponding orbital period changes (dot-dashed line).

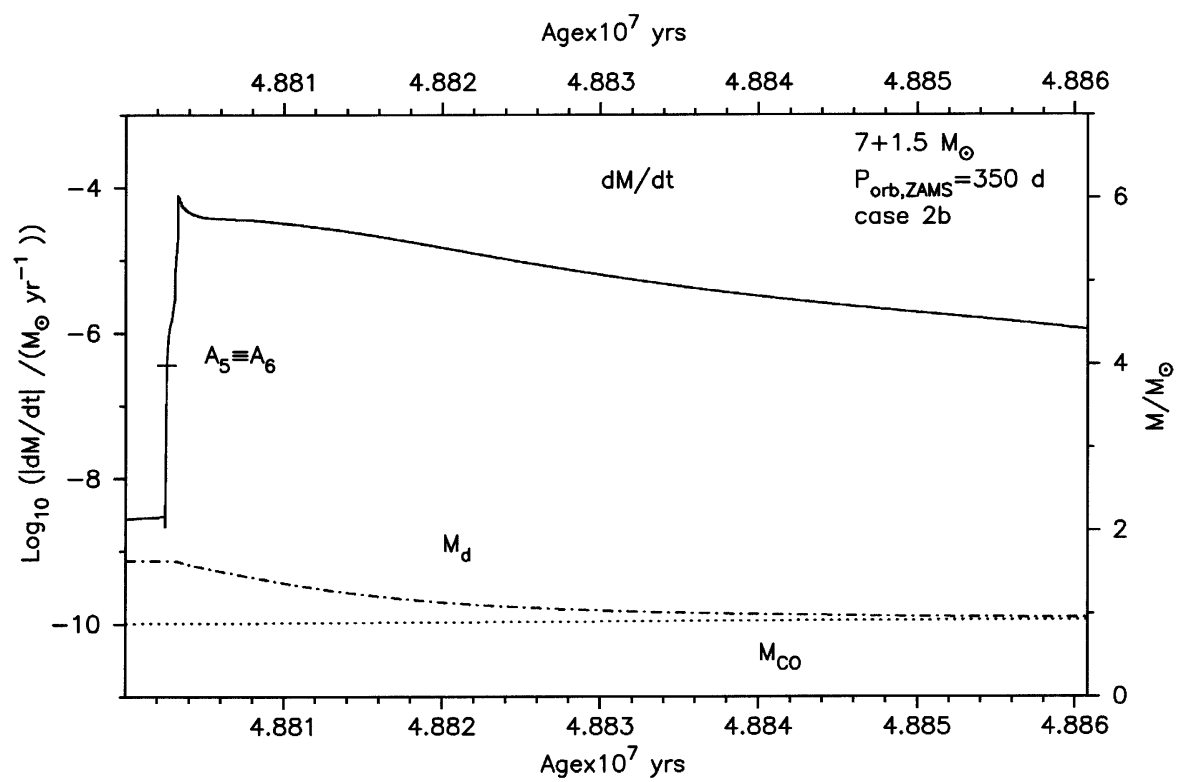

Fig. 4. As Fig. 2 (lower) but for case 2b. Here the time variation of $\dot{M}$ up to point $\mathrm{A}_{5}$ is the same as in the upper panel of Fig. 2, for case $2 \mathrm{a}$. However, in this case of evolution we assume tidally enhanced winds to dominate at $A_{5}$ so that they prevent the post-CE primary to overfill its Roche lobe after CE evolution. As a result, matter can not be accreted on the secondary, which now retains its solar composition.

added to the $\mathrm{CO}$ core. The core mass increases and at the end of Roche lobe overflow, $3.7 \times 10^{5} \mathrm{yr}$ after the end of the CE phase (point $\mathrm{A}_{6}$ in Fig. 1), the core mass has reached $M_{\mathrm{CO}}=0.98 M_{\odot}$. The total mass of the remnant is then $1.05 M_{\odot}$. If mass loss driven by radiative winds is weak this donor maintains contact with its Roche lobe under the intense shell burning. The semidetached phase itself lasts for about $5.6 \times 10^{4} \mathrm{yr}$ during which a total mass of $0.6 M_{\odot}$ is transferred to the secondary.

In case $2 b$ the remnant avoids overflowing its Roche lobe for a second time because enhanced mass loss strips its envelope before it expands. From points $A_{6}-A_{7}$ in Fig. 1 the evolution is dominated by the enhanced mass loss. The star moves to the blue and reaches a maximum temperature of $T_{\text {eff }}=10^{5.48} \mathrm{~K}$. It develops a degenerate $\mathrm{CO}$ core of $0.98 M_{\odot}$ with a very thin $\mathrm{He}$ rich envelope of $3.0 \times 10^{-3} M_{\odot}$, about $3.9 \times 10^{5} \mathrm{yr}$ after the first EAGB Roche lobe overflow.

From point $\mathrm{A}_{7}$ onwards the donor enters the white dwarf cooling nose after the exhaustion of its nuclear fuel. Its radius contracts to less than $10^{-2} R_{\odot}$ with a surface temperature of $T_{\text {eff }} \approx 10^{4} \mathrm{~K}$ and luminosity $L=10^{-3.37} L_{\odot}$. The final white 


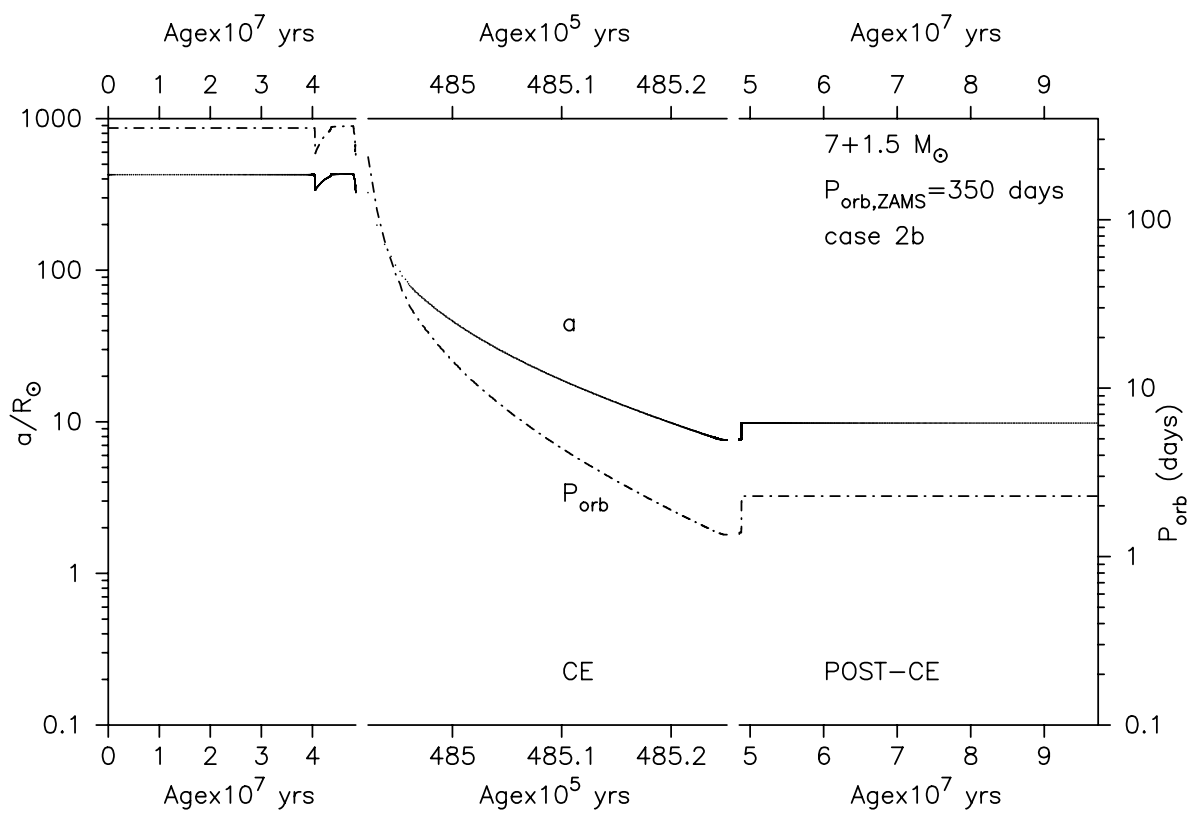

Fig. 5. As Fig. 3 but for case $2 b$.

dwarf has a degenerate $\mathrm{CO}$ core of $0.977 M_{\odot}$ and a very thin He atmosphere (about $1 \times 10^{-4} M_{\odot}$ ). The final orbital period is $2.04 \mathrm{~d}$ and the companion mass is $2.1 M_{\odot}$.

\section{Evolution of the secondary component}

For case $2 \mathrm{a}$ we assume that the star experiences thermohaline mixing, a slow instability which mixes up the added matter in the non-convective regions of the envelope of the companion star (Kippenhahn 1980; Veronis 1963). This should be so whenever the diffusion timescale is considerably shorter than the thermal time scale. For the case of a $15 M_{\odot}$ main-sequence star accreting $1.0 M_{\odot}$ of He the diffusion timescale is of the order of $10^{4} \mathrm{yr}$ and the added material mixes in before any thermal response. In our case we conjecture that thermohaline mixing effectively mixes the added $\mathrm{He}$ in the envelope of the secondary: we approximate this by calculating a ZAMS model with total mass of $2.1 M_{\odot}$ of homogeneous chemical composition of $X_{\mathrm{H}}=0.5$, and $X_{\mathrm{He}}=0.48$, because the initial $1.5 M_{\odot}$ secondary (of solar composition) has accreted $0.6 M_{\odot}$ of helium rich matter. We evolve a binary consisting of this $2.1 M_{\odot}$ star with a WD companion of $1 M_{\odot}$ in an orbit of $1.4 \mathrm{~d}$. We find that the secondary can transfer mass at sufficiently high rates during Roche lobe overflow, on the MS and while crossing the Hertzsprung gap, for the WD to grow to produce a SN Ia. In the meantime it would appear as a SSS. Such evolution is illustrated in Fig. 6. The top panel shows the variation of the WD mass as a function of time (dashed line) while the full line is the orbital period. In the lower panel the full line depicts the mass transfer rates which produce growth of the white dwarf as a result of nuclear burning. The dotted lines indicate the boundaries of the region of steady nuclear burning on the WD. However, another possibility for case-2a evolution could arise if the diffusion timescale is longer than the thermal timescale and thermohaline mixing is negligible. In this case a second CE phase or the formation of a contact binary could occur. This has been studied for example by Neo et al. (1977), Iben \& Livio (1993). Such a system would merge.

In case $2 \mathrm{~b}$ the binary system consists of a $1.5 M_{\odot}$ MS star of solar composition plus a CO WD of $0.94 M_{\odot}$. From Fig. 7 (lower) we conclude that the secondary cannot sustain thermaltimescale mass-transfer rates and so the accreted hydrogen burns unsteadily on the WD surface. When mass-transfer rates are lower than $3 \times 10^{-8} M_{\odot} \mathrm{yr}^{-1}$ hydrogen burns unsteadily in strong novae and the ensuing erosion of the WD makes it very improbable that it will ever reach the Chandrasekhar mass. Another evolutionary fate of these systems might be that, during the strong nova outburst a CE could result from rapid envelope expansion driven by a rapid nuclear energy injection leaving a double white dwarf system (Iben \& Livio 1993). If these merge in a Hubble time and the sum of their masses exceeds the Chandrasekhar limit a NS may be formed (Hachisu 2002) while if the total mass of both WDs combined is below Chandrasekhar limit a merged WD can be formed. Such massive WDs have been reported by Green et al. (2000).

Results for different donor masses can be summarised as follows. For case 2 a evolution secondaries of 1.7, 2.0, 2.5 and 3.0 $M_{\odot}$ can accrete around $0.6 M_{\odot}$ during shell He burning of the primary and increase their mass in a way similar to a donor of $1.5 M_{\odot}$. For these systems, Roche lobe overflow from the secondary to the WD is the dominant mechanism for masstransfer. Higher donor masses of 3.1 and 3.6 $M_{\odot}$ can support transfer rates higher than $10^{-5} M_{\odot} \mathrm{yr}^{-1}$ beyond which there are no theoretical calculations for the mass accumulation efficiency parameter in helium shell flashes (Kato \& Hachisu 1999).

\section{Concluding results for $\mathrm{CE}$ evolution}

Common envelope evolution for the general case of a $7 M_{\odot}$ primary is presented in Figs. 8-10. Figure 8 shows the 

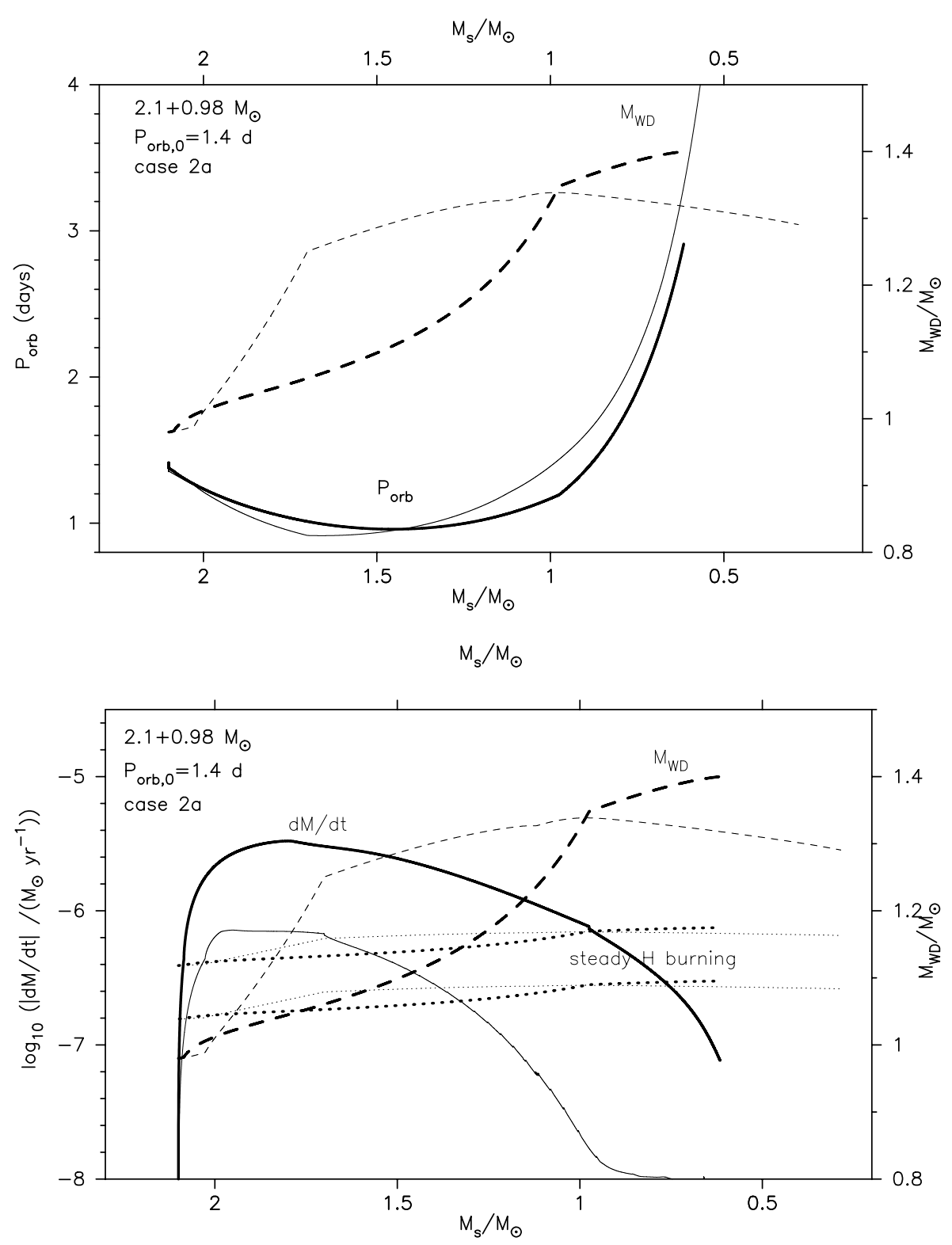

Fig. 6. Case-2a evolution for a post-CE binary with an He enriched donor of $2.1 M_{\odot}$. The companion is a $0.98 M_{\odot} \mathrm{CO}$ white dwarf at an orbital period $P_{\text {orb }} \approx 1.4 \mathrm{~d}$. Upper panel: variation of $P_{\text {orb }}$ (thick solid line) and $M_{\mathrm{WD}}$ (thick dashed line) as a function of the secondary mass $M_{\mathrm{s}}$. Lower panel: evolution of $\dot{M}$ (thick solid line) and $M_{\mathrm{WD}}$ (thick dashed line) versus secondary mass. The system is a candidate luminous SSS and possibly a SN Ia. For comparison the evolution of a $2.1 M_{\odot}$ solar composition donor is shown with thin lines. In this case the WD does not grow sufficiently to become a SN Ia. The nearly horizontal dotted lines define the region of steady H burning, thick for our case $2 \mathrm{a}$ and thin for solar composition.

variation of the binding energy of the envelope as a function of time for both definitions of core boundaries just inside the $\mathrm{H}$ burning shell, $\left(M=M_{\mathrm{H}}\right)$, and just inside the He-burning shell $\left(M=M_{\mathrm{He}}\right)$. Figure 9 shows the final binary separation and final orbital period as a function of the initial parameters (orbital separation and binary period) at the onset of Roche lobe overflow for all radii for which the star develops deep convective envelope. The range of the radius corresponding to the whole extent of EAGB phase is also indicated with vertical dashed lines. For the $1.5 M_{\odot}$ companion the CE phase begins when the donor's radius is $167 R_{\odot}$ and the orbital separation is $325 R_{\odot}$. Figure 10 shows the minimum $\alpha_{\mathrm{CE}}$ parameter for which systems can survive as binaries for both definitions of core boundary. When the core boundary is taken inside the Heburning shell, systems rarely survive the CE.

Our main conclusion here is that the appropriate binding energy of the envelope for CE evolution should be integrated from the hydrogen-burning shell outwards. If it is calculated from the helium-burning shell the cores are much less likely to survive detached.

\section{Comparison with observations}

The calculations of CE evolution can be compared with observations of bright subdwarf systems such as HD49798 which has a pulsing X-ray companion (Bisscheroux et al. 1997) and is 

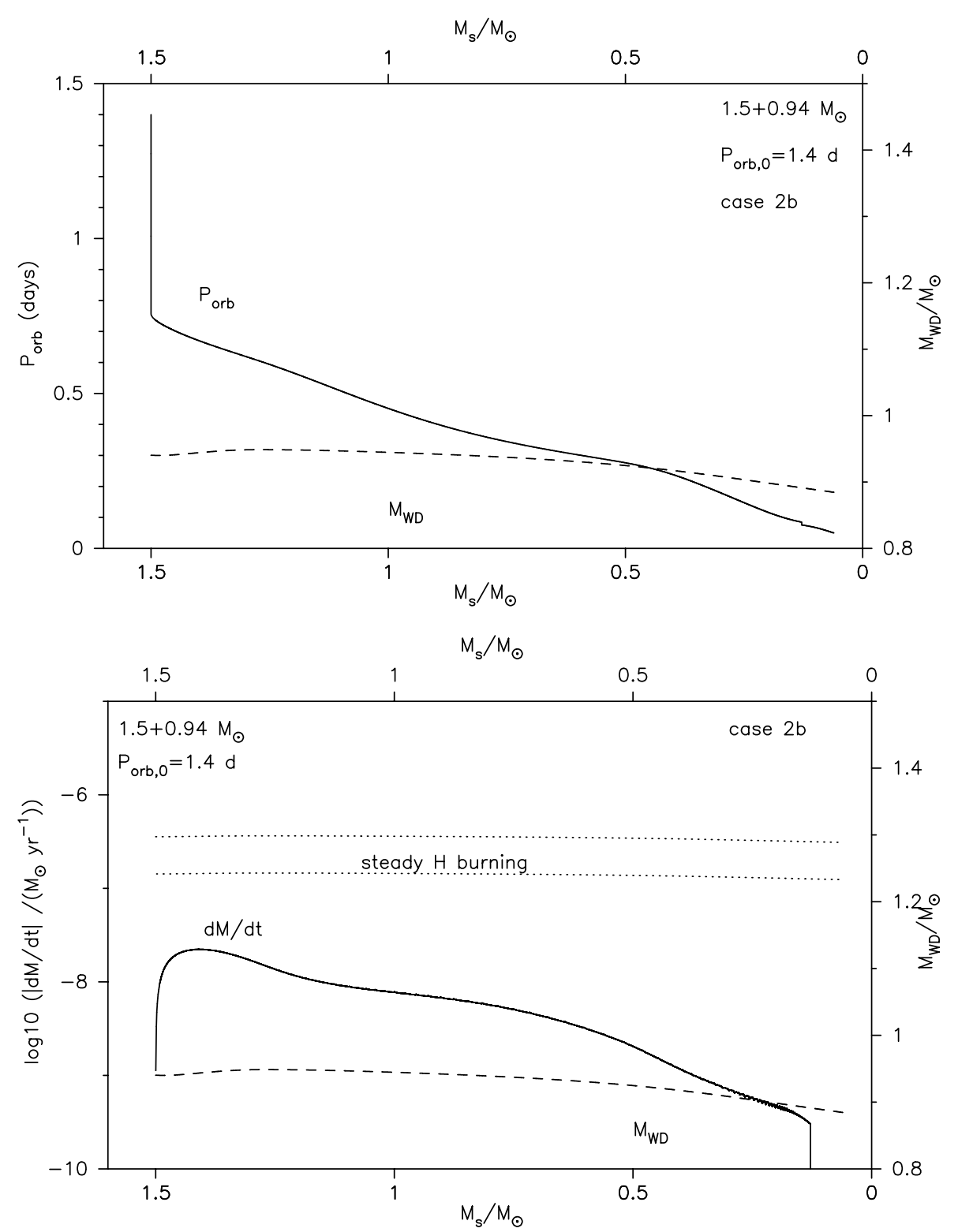

Fig. 7. As Fig. 6 but for case 2b. The post-CE companion maintains its solar composition and mass of $1.5 M_{\odot}$ with a $0.94 M_{\odot}$ CO white dwarf. Initially $P_{\text {orb }} \approx 1.4 \mathrm{~d}$. This system does not evolve as a SSS and is not a potential candidate for a SN Ia.

plotted in the top panel of Fig. 1. The track labelled B in Fig. 1 shows the evolution of the secondary component for cases $2 \mathrm{a}$ and $2 \mathrm{~b}$. For comparison we have also plotted the position of the system HS $0705+6700$ in the HR diagram according to recent observations by Drechsel et al. (2000). Our computed CE evolution can be compared with this system which appears to have attained thermal equilibrium.

Our evolutionary calculations for SSSs can be compared with observations as follows:

i) Super-Eddington extragalactic SSSs

Although the number of SSSs and their spatial distribution in galaxies, such as M 31 and M 81, detected by CHANDRA are globally consistent with theoretical predictions (Di Stefano \& Rappaport 1994; Yungelson 1996, 1998) there are a few sources with X-ray properties which correspond to bolometric luminosities exceeding the Eddington limit for spherical accretion on to a $1.4-M_{\odot}$ star (Swartz et al. 2002).
For example the source (N1) in M 81 has a bolometric luminosity that can only be explained with accretion rates of the order of $5 \times 10^{-6} M_{\odot} \mathrm{yr}^{-1}$. A possible model is a thick wind from a massive WD (Hachisu et al. 1996). At the required accretion rates, without such a wind, the photosphere of the WD would expand to red giant dimensions and no X-rays would be detected. On the other hand if the burning triggers a strong opaque wind then, as the accretion rate temporarily decreases, the mass-loss rate and the photospheric radius decrease exposing regions near the burning shell and temporarily a very high Super-Eddington X-ray luminosity may be observed. In any case a very massive WD is required and this fits well with our $7-M_{\odot}$ progenitor.

\section{ii) U Scorpii}

Another interesting case is the, presumably helium-rich, SSS U Sco. Its effective blackbody temperature is $9 \times 10^{5} \mathrm{~K}$ equivalent to $k T \approx 0.2-2 \mathrm{keV}$ and so at its estimated 


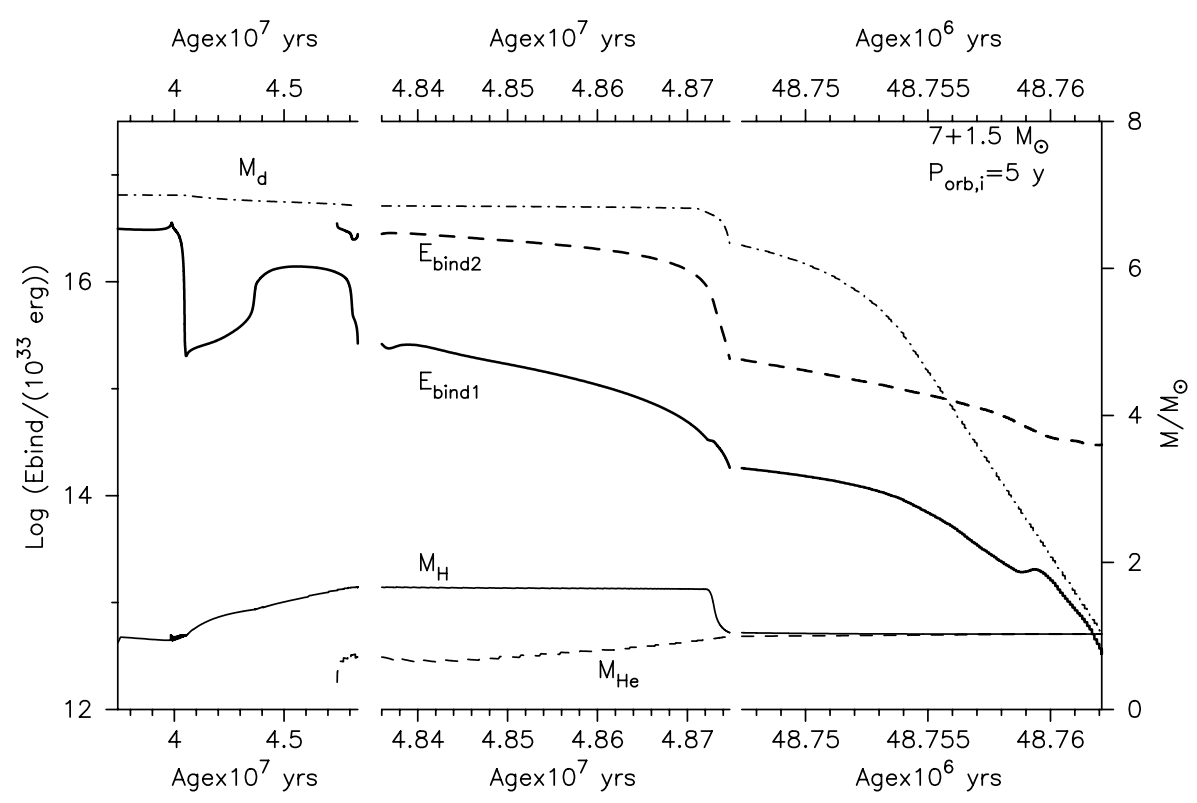

Fig. 8. $E_{\text {bind }}$ (left axis) and donor's mass (right hand axis) versus time. Indices 1 and 2 denote the two core definitions inside the H-shell $\left(M=M_{\mathrm{H}}\right)$ and inside He-shell burning $\left(M=M_{\mathrm{He}}\right)$. Note that the time axis is different for the pre-AGB, EAGB and TPAGB.

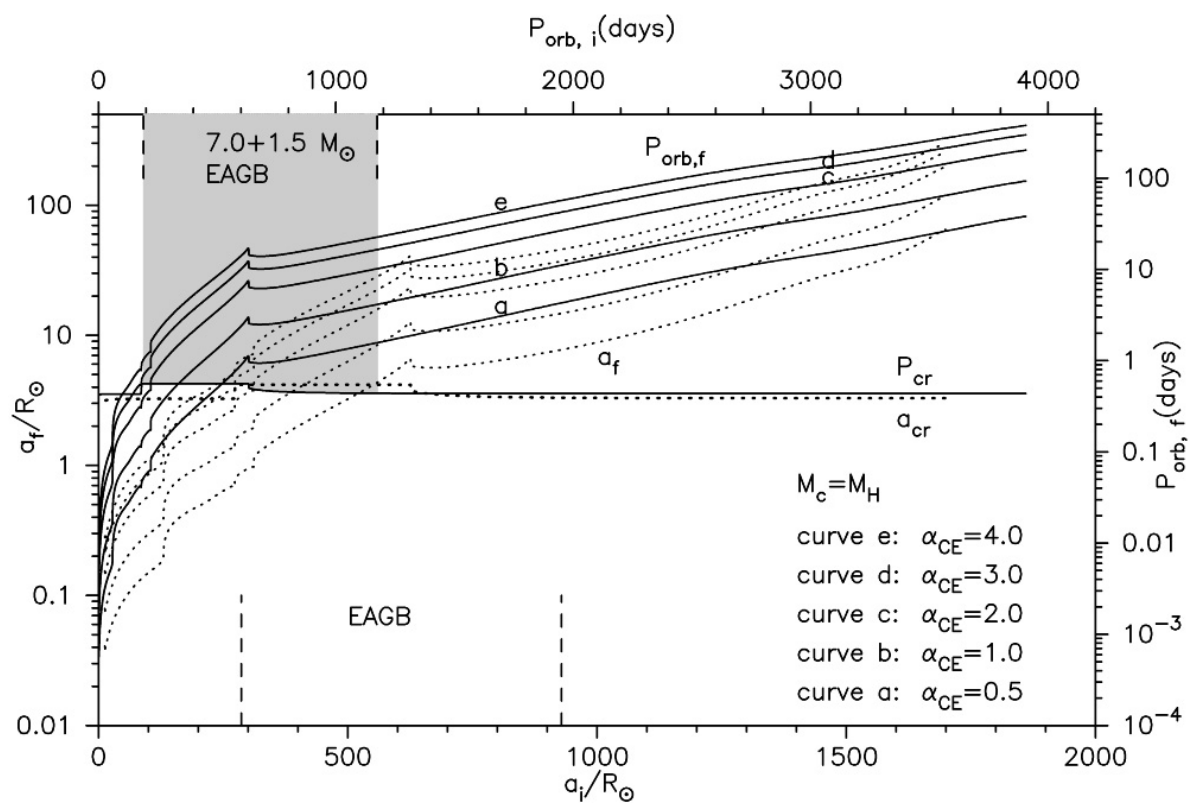

Fig. 9. Final orbital separation after the CE phase (dotted line) as a function of the separation at the onset of the CE phase (left and lower axes). Solid lines are orbital periods (right and upper axes). Results are for five different values of $\alpha_{\mathrm{CE}}$ from 0.5-4.0. Vertical dashed lines show the boundaries of EAGB evolution. The core mass is the mass inside the H-burning shell $M_{\mathrm{H}}$. The critical orbital separation $a_{\mathrm{cr}}$ and period $P_{\mathrm{cr}}$ at which the companion star fills its Roche lobe at the end of the CE phase are also given. Systems within the grey area satisfy our criterion for survival of the binary system as described in Sect. 3.1.2.

distance of about $10-14 \mathrm{kpc}$, its bolometric luminosity is in the range $(0.6-2.4) \times 10^{38} \mathrm{erg} \mathrm{s}^{-1}$ (Kahabka et al. 1999). From the B light curve fit the donor is a MS star of $1.5 M_{\odot}$ although the possibility of a MS donor with mass anywhere in the range $0.8-2.0 M_{\odot}$ can not be excluded (Hachisu et al. 2000a, $2000 \mathrm{~b}$ ). From the same data the mass of the WD is $1.37 M_{\odot}$, rather near the Chandrasekhar limit. Optical and UV spectra of the ejecta reveal that the helium abundance is high. By number of atoms it ranges from $N(\mathrm{He}) / N(\mathrm{H})=0.16 \pm 0.02$ (Iijima 2002) to about 2 . The higher value is at the maximum of the 1979 outburst (Barlow et al. 1981). The orbital period of the system is $1.23 \mathrm{~d}$ (Schaefer \& Ringwald 1995) within the range of our presented calculations (see the top panel of Fig. 4) for either case. For example, following in detail the evolution of a progenitor binary of $7+1.5 M_{\odot}$ from the ZAMS, as in Figs. 1 and 2, we end up with a $2.1 M_{\odot}$ donor and a CO WD of mass $0.98 M_{\odot}$ in an orbital period of $1.4 \mathrm{~d}$ (Fig. 4). If the He abundance enhancement is real then case-2a evolution may account for the formation and evolution of U Sco. The final outcome of this system will be the formation of a SSS which 

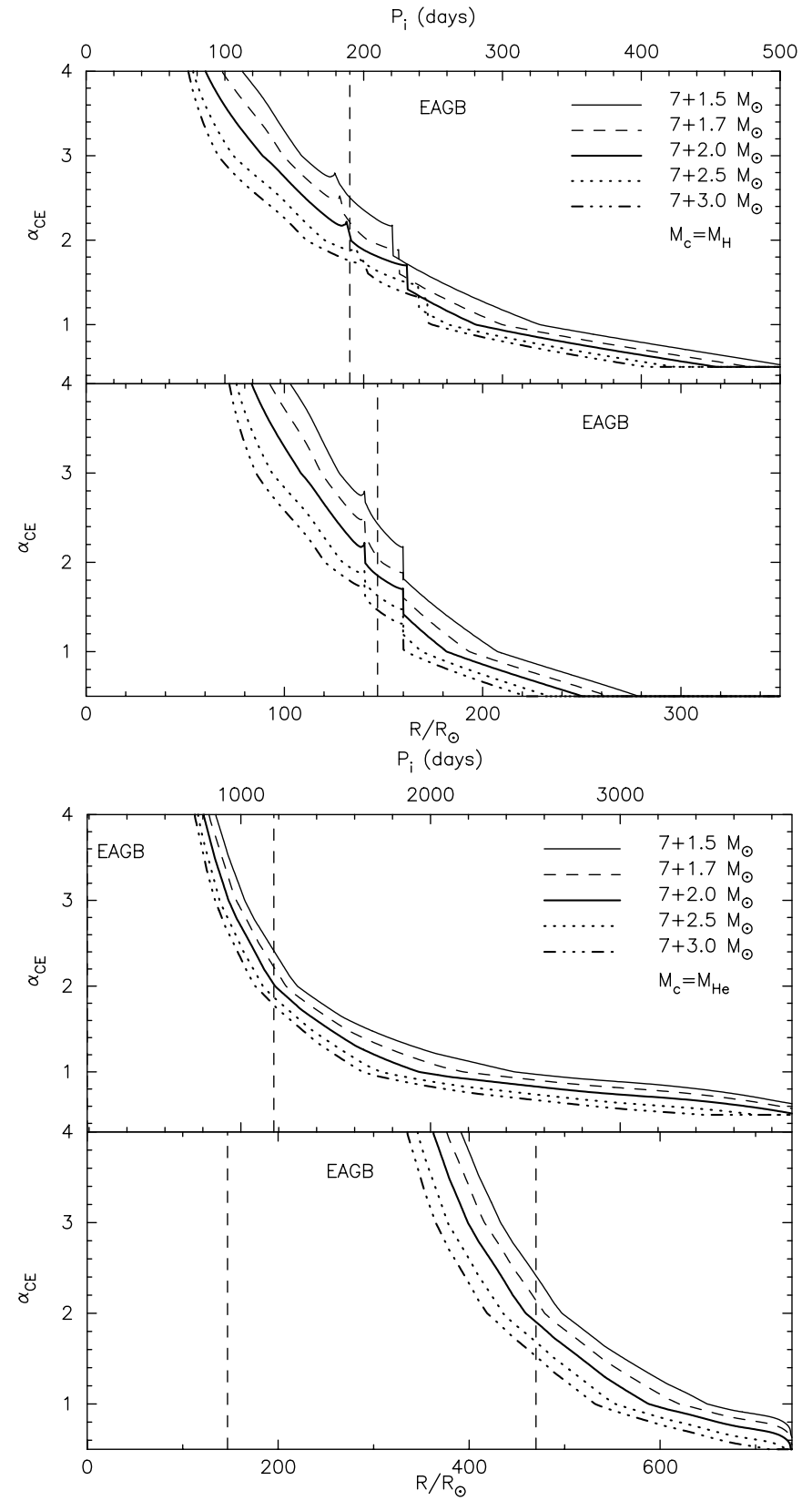

Fig. 10. Upper panel: minimum CE efficiency parameters $\alpha_{\mathrm{CE}}$ when the core is just inside the H-burning shell $M_{\mathrm{c}}=M_{\mathrm{H}}$. Dashed vertical lines indicate the boundaries of EAGB evolution. Lower panel: minimum $\alpha_{\mathrm{CE}}$ when the core boundary lies just inside He-burning shell $M_{\mathrm{c}}=M_{\mathrm{He}}$.

may lead to a SN Ia explosion because the WD can grow to the Chandrasekhar limit (see Fig. 4). Without winds the duration of the steady burning phase is about $5 \times 10^{5} \mathrm{yr}$ (Fig. 4 , lower panel) and the calculated bolometric luminosity would range from about 0.6 to $1.5 \times 10^{38} \mathrm{erg} \mathrm{s}^{-1}$ (from $L_{\mathrm{Bol}}=X \epsilon \dot{M}_{\mathrm{H}}$, with $\epsilon=\epsilon_{\mathrm{H}}=6 \times 10^{18} \mathrm{erg} \mathrm{g}^{-1}$ and $\dot{M}_{\mathrm{H}}$ the required rate for steady $\mathrm{H}$ burning). This is longer than obtained by Hachisu et al. (1999) by a factor of 5/3 because we have used a different critical accretion rate for triggering thick winds from the WD. We consider steady $\mathrm{H}$ burning while they take the critical rate for steady He burning because they assume a He-star companion. If, on the other hand, we work with solar abundances for the secondary then, for a $2.1-M_{\odot}$ donor at the same orbital separation, the SSS phase lasts longer, about $8 \times 10^{5} \mathrm{yr}$. Figure 4 shows that the helium enhanced donor of the supersoft source (case 2a), can increase the white dwarf mass to the observed value $1.37 M_{\odot}$ (Hachisu et al. 2000a, 2000b), while the solar-abundance donor cannot. So classical donors for U Sco do not drive a SSS which is a candidate SN Ia. This confronts theory with observations (Branch 1995). We conclude that, because the companion of $\mathrm{U}$ Sco is helium rich, this system is a good candidate for evolution to a SN Ia.

\section{iii) CAL 83}

Our $P_{\text {orb }}$ and mass of the secondary are also in agreement with those of the luminous supersoft source CAL83 which is considered a prototypical supersoft X-ray source. It has an orbital period of $1.04 \mathrm{~d}$ and the mass of its white dwarf exceeds $1.3 M_{\odot}$ (Alcock et al. 1997). The radius of the WD, deduced from its spectrum, is about $10^{9} \mathrm{~cm}$ so it is in a slightly expanded state (Kahabka 1998). Such occasional bloating could be explained by unstable nuclear burning on a massive WD caused by high accretion rates close to $10^{-6} M_{\odot} \mathrm{yr}^{-1}$ (Southwell et al. 1997; Kahabka 1996). An alternative explanation would be an episodic mass transfer from the donor owing to magnetic star spot activity near the $L_{1}$ point (Kahabka 1998). Strong winds and a possible jet have also been observed, indicative of the presence of an unstable disc owing to high luminosities from the central source (Southwell et al. 1997).

\section{Conclusions}

We have presented a new set of computations for the formation of SSSs in which thermohaline mixing is taken into account. This process is shown to modify the conventional evolution in which donors are of their primordial chemical composition throughout the evolution. We note that the change of mass and chemical composition is a physical consequence of the interaction between the binary members and is supported by the observed characteristics of systems such as U Sco.

Our main conclusions can be summarised as follows:

- Case-2a evolution considers non-solarlike donors in the SSSs with mass ranging from $2.1-2.4 M_{\odot}$.

- Case-2b evolution considers more massive solar-like donors (e.g. $M_{\mathrm{s}} \approx 2.5 M_{\odot}$ ).

- Such case-2a evolution significantly modifies the binary evolution and leads to more rapid nuclear evolution of the SSS donor (Bitzaraki et al. 2003a).

- During He shell burning the post-CE remnant of the primary can transfer up to $0.6 M_{\odot}$ to the secondary.

- Winds prove to be an essential element in the binary evolution and must be treated as realistically as possible.

- We have examined two definitions for the boundary of the core, just inside H-burning shell and just inside the He-burning shell. Systems would be unlikely to survive $\mathrm{CE}$ evolution if the core boundary were inside the Heburning shell. When we model the CE phase by rapid mass loss we find that the primary shrinks within its Roche lobe as soon as the H-burning shell is exposed. 
- Standard CE evolution demands that the efficiency parameter $\alpha_{\mathrm{CE}} \geq 3$ if the binary system is to survive.

- Enhanced superwinds facilitate the cooling of the WD during post-CE evolution.

- New observations seem to verify the existence of SSSs with enhanced He abundances similar to those we find in case $2 \mathrm{a}$ evolution.

Acknowledgements. We thank the anonymous referee for helping to improve the original manuscript. We also thank Dr. Savonije for his contribution to the binary subroutines implemented in the Eggleton code. OB wishes to express her gratitude and thanks to the Hellanic Ministry of Education for providing leave of absence from her job. She would like to thank Astronomical Institute of Amsterdam for extensive access to their computer facilities and for financial support during her stay there and the University of Athens (Grant No. 70/4/3305) and the British Council for supporting her visit to the Institute of Astronomy in Cambridge. CAT thanks Churchill college for a fellowship. OB also thanks Dr. T. Blöcker (Max-Planck-Institut für Radioastronomie), Prof. I. Hachisu (Keio University, Japan), Prof. M. Livio (Space Telescope Science Institute), Prof. P. Wood (Australian National University), and Dr. L. Yungelson (Russian Academy of Sciences), for electronically sending her one of their publications and fruitful discussions.

\section{References}

Alcock, C., Allsman, R. A., Alves, D., et al. 1997, MNRAS, 286, 483 Alexander, D. R., \& Ferguson, J. W. 1994a, in Molecules in the Stellar Environment, ed. U. G. Jorgensen (Berlin: Springer-Verlag), 149 Alexander, D. R., \& Ferguson, J. W. 1994b, ApJ, 437, 879

Barlow, M. J., Brodie, J. P., Mayo, S. K., et al. 1981, MNRAS, 195 , 61

Bitzaraki, O. M., Tout, C., \& Rovithis-Livaniou, H. 2003a, New Astron., 8, 23

Bitzaraki, O. M., van den Heuvel, E. P. J., Tout, C., \& Rovithis-Livaniou, H. 2003b, Interplay of Periodic, Cyclic and Stochastic Variability in Selected Areas of the H-R Diagram, ed. C. Sterken, ASP Conf. Ser., 292 (Astronomical Society of the Pacific), 241

Bisscheroux, B. C., Pols, O. R., Kahabka, P., Belloni, T., \& van den Heuvel, E. P. J. 1997, A\&A, 317, 815

Blöcker, T. 1995, A\&A, 297, 727

Bowen, G. H. 1988, ApJ, 329, 299

Branch, D., Livio, M., Yungelson, L. R., Boffi, F. R., \& Baron, E. 1995, PASP, 107, 1019

Carraro, G. 1996, Ph.D. Thesis, Padova Univ.

Caughlan, G. R., Fowler, W. A., Harris, M. J., \& Zimmerman, B. A. 1985, At. Data Nucl. Data Tables 32, 197

Caughlan, G. R., \& Fowler, W. A. 1988, At. Data Nucl. Data Tables 40, 283

de Jager, C., Neeuwenhuijzen, H., \& van der Hucht, K. A. 1988, A\&AS, 72, 259

de Kool, M. 1990, ApJ, 358, 189

Dewi, J. D. M., \& Tauris, T. M. 2000, A\&A, 360, 1043

Di Stefano, R., \& Rappaport, S. 1994, ApJ, 437, 733

Drechsel, H., Heber, U., Napiwotzki, R., et al. 2001, A\&A, 379, 893

Eggleton, P. P. 1971, MNRAS, 151, 351

Eggleton, P. P. 1972, MNRAS, 156, 361

Eggleton, P. P. 1983, MNRAS, 268, 368

Eggleton, P. P., Faulkner, J., \& Flannery, B. P. 1973a, A\&A, 23, 325

Eggleton, P. P. 1973b, MNRAS, 163, 279
Fujimoto, M. Y. 1982a, ApJ, 257, 767

Fujimoto, M. Y. 1982b, ApJ, 257, 752

Greiner, J. 2002, ApJ, 578, L59

Greiner, J. 2000, New Astron., 5, 137

Greiner, J. 1996, in Supersoft X-Ray Sources, ed. J. Greiner, Lecture Notes in Physics (New York: Springer), 472, 75

Green, P. J., Ali, B., \& Napiwotzki, R. 2000, ApJ, 540, 992

Hamann, W. R., \& Koesterke, L. 1998, A\&A, 335, 1003

Hamann, W. R., Koesterke, L., \& Wessolowski, U. 1995, A\&A, 299, 151

Han, Z., Podsiadlowski, P., \& Eggleton, P. P. 1994, MNRAS, 270, 121

Hachisu, I. 2002, The Physics of Cataclysmic Variables and Related Objects, ed. B. T. Gänsicke, K. Beuermann, \& K. Reinsch, ASP Conf. Ser., 261, 627

Hachisu, I., Kato, M., Kato, T., Matsumoto, K., \& Nomoto, K. 2000a, ApJ, 534, L189

Hachisu, I., Kato, M., Kato, T., \& Matsumoto, K. 2000b, ApJ, 528, L97

Hachisu, I., Kato, M., Nomoto, K., \& Umeda, H. 1999, ApJ, 519, 314

Hachisu, I., Kato, M., \& Nomoto, K. 1996, ApJ, 470, L97

Humphreys, R. M., \& Davidson, K. 1994, PASP, 106, 1025

Hurley, J. R., Pols, O. R., \& Tout, C. A. 2000, MNRAS, 315, 543

Iben, I. J., \& Livio, M. 1993, PASP, 105, 1373

Iben, I. J., \& Renzini, A. 1983, ARA\&A, 21, 271

Iijima, T. 2002, A\&A, 387, 1013

Itoh, N., Adachi, T., Nakagawa, M., Kohyama, Y., \& Munakata, H. 1989, ApJ, 339, 354

Itoh, N., Mutoh, H., Hikita, A., \& Kohyama, Y. 1992, ApJ, 395, 622

Itoh, N., Hayashi, H., Nishikawa, A., \& Kohyama, Y. 1996, ApJS, 102,411

Kahabka, P. 1998, A\&A, 331, 328

Kahabka, P. 1996, in Supersoft X-Ray Sources, ed. J. Greiner, Lecture Notes in Physics (New York: Springer), 472, 215

Kahabka, P., \& van den Heuvel, E. P. J. 1997, ARA\&A, 35, 69

Kahabka, P., Hartmann, H. W., Parmar, A. N., \& Negueruela, I. 1999, A\&A, 347, L43

Kato, M., \& Hachisu, I. 1999, ApJ, 513, L41

Kato, M., Saio, H., \& Hachisu, I. 1989, ApJ, 340, 509

Kippenhahn, R., Ruschenplatt, G., \& Thomas, H.-C. 1980, A\&A, 91, 175

Knapp, G. R., \& Morris, M. 1985, ApJ, 292, 640

Kudritzki, R. P., Pauldrach, A., Puls, J., \& Abbott, D. C. 1989, A\&A, 219, 205

Kudritzki, R. P., \& Reimers, D. S. 1978, A\&A, 70, 227

Landau, L. D., \& Lifshitz, E. M. 1965, Quantum mechanics

Neo, S., Miyaji, S., Nomoto, K., \& Sugimoto, D. 1977, PASJ, 29, 249

Nieuwenhuijzen, H., \& de Jager, C. 1990, A\&A, 231, 134

Nomoto, K. 1982, ApJ, 253, 798

Pols, O. R., Schröder, K.-P., Hurley, J. R., Tout, C. A., \& Eggleton, P. P. 1998, MNRAS, 298, 525

Pols, O. R., Tout, C. A., Eggleton, P. P., \& Han, Z. 1995, MNRAS, 274, 964

Prialnik, D., \& Kovetz, A. 1995, ApJ, 445, 789

Rappaport, S., Joss, P. C., \& Verbunt, F. 1983, ApJ, 275, 713

Regös, E., \& Tout, T. M. 1995, Cape Workshop on Magnetic Cataclysmic Variables, ASP Conf. Ser., 85, 458

Rogers, F. J., \& Iglesias, C. A. 1992, ApJS, 79, 507

Schaefer, B. E., \& Ringwald, F. A. 1995, ApJ, 447, L45

Soberman, G. E., Phinney, E. S., \& van den Heuvel, E. P. J. 1997, 327 , 620

Southwell, K. A., Livio, M., \& Pringle, J. E. 1997, ApJ, 478, L29

Southwell, K. A., Livio, M., Charles, P. A., O’Donoghue, D., \& Sutherland, W. J. 1996, ApJ, 470, 1065 
Swartz, D. A., Ghosh, K. K., Suleimanov, V., Tennant, A. F., \& Wu, K. 2002, ApJ, 574, 382

Taam, R. E., \& Sandquist, E. L. 2000, ARA\&A, 38, 113

Taam, R. E. 1996, in Compact Stars in Binaries, ed. J. van Paradijs,

E. P. J. van den Heuvel, \& E. Kuulkers (Dordrecht: Kluwer Acad. Publ.), 165, 3

Tout, C. A., Pols, O. R., Eggleton, P. P., \& Han, Z. 1996, MNRAS, 281,257

Tout, C. A., \& Hall, D. S. 1991, MNRAS, 253, 9
Vassiliadis, E., \& Wood, P. R. 1993, ApJ, 413, 641

van den Heuvel, E. P. J., Bhattacharya, D., Nomoto, K., \& Rappaport, S. A. 1992, A\&A, 262, 97

Veronis, G. J. 1963, ApJ, 137, 641

Webbink, R. F. 1984, ApJ, 277, 355

Weidemann, V. 2000, A\&A, 363, 647

Yungelson, L., \& Livio, M. 1998, ApJ, 497, 168

Yungelson, L., Livio, M., Truran, J. W., Tutukov, A., \& Fedorova, A. 1996, ApJ, 466, 890 